\title{
A novel maize gene, glossy6 involved in epicuticular wax deposition and drought tolerance
}

Li LI ${ }^{1,2,3, \dagger}$, Yicong Du ${ }^{1, \dagger}$, Cheng He ${ }^{1,4}$, Charles R. Dietrich ${ }^{2,5}$, Jiankun $\mathrm{Li}^{1}$, Xiaoli Ma ${ }^{6,7}$, Rui Wang ${ }^{1}$, Qiang

Liu $^{2,6,}$ Sanzhen Liu ${ }^{2,4}$, Guoying Wang ${ }^{1}$, Patrick S Schnable ${ }^{2,6, *}$, Jun Zheng,"*

1 Institute of Crop Sciences, Chinese Academy of Agricultural Sciences, Beijing 100081, P.R. China

2 Department of Agronomy, Iowa State University, Ames, IA 50011-3650, USA

3 Current address: Seed Science and Technology Research Center, Beijing Innovation Research Center on the Whole Process of Crop Seeds, China Agricultural University, Beijing 100193, China.

4 Department of Plant Pathology, Kansas State University, Manhattan KS 66506, USA

5 Current address: Monsanto, Chesterfield, Missouri, 63005-63017, USA

6 College of Agronomy and Biotechnology, China Agricultural University, Beijing 100193, China

7 Current address: Center for Plant Molecular Biology, University of Tübingen, Tübingen 72076, Germany

tThese authors contributed equally to this article.

*For correspondence: zhengjun02@caas.cn and schnable@iastate.edu

RUNNING TITLE:

gl6 involved in maize epicuticular wax deposition 


\section{SUMMARY}

Epicuticular waxes, long-chain hydrocarbon compounds, form the outermost layer of plant surfaces in most terrestrial plants. The presence of epicuticular waxes protects plants from water loss and other environmental stresses. Cloning and characterization of genes involved in the regulation, biosynthesis, and extracellular transport of epicuticular waxes on to the surface of epidermal cells have revealed the molecular basis of epicuticular wax accumulation. However, intracellular trafficking of synthesized waxes to the plasma membrane for cellular secretion is poorly understood. Here, we characterized a maize glossy $(g l 6)$ mutant that exhibited decreased epicuticular wax load, increased cuticle permeability, and reduced seedling drought tolerance relative to wild type. We combined an RNA-sequencing based mapping approach (BSR-Seq) and chromosome walking to identify the gl6 candidate gene, which was confirmed via the analysis of multiple independent mutant alleles. The gl6 gene represents a novel maize glossy gene containing a conserved, but uncharacterized domain. Functional characterization suggests that the GL6 protein may be involved in the intracellular trafficking of epicuticular waxes, opening a door to elucidating the poorly understood process by which epicuticular wax is transported from its site of biosynthesis to the plasma membrane.

Keywords: maize (Zea mays), glossy mutant, epicuticular waxes, drought tolerance, DUF538 


\section{SIGNIFICANCE STATEMENT}

Plant surface waxes provide an essential protective barrier for terrestrial plants. Understanding the composition and physiological functions of surface waxes, as well as the molecular basis underlying wax accumulation on plant surfaces provides opportunities for the genetic optimization of this protective layer. Genetic studies have identified genes involved in wax biosynthesis, extracellular transport, as well as spatial and temporal regulation of wax accumulation. In this study, a maize mutant, gl6 was characterized that exhibited reduced wax load on plant surfaces, increased water losses, and reduced seedling drought tolerance compared to wild type controls. The gl6 gene is a novel gene harboring a conserved domain with an unknown function. Quantification and microscopic observation of wax accumulation as well as subcellular localization of the GL6 protein provided evidence that $g l 6$ may be involved in the intracellular trafficking of waxes, opening a door for studying this necessary yet poorly understood process for wax loading on plant surfaces. 


\section{INTRODUCTION}

The hydrophobic cuticle covers most aerial parts of land plants, which acts as a barrier to protect plants from non-stomatal water loss, ultraviolet (UV) light, physical damage caused by insects or fungi, and from other biotic or abiotic stresses (Shepherd and Wynne Griffiths, 2006). The cuticle mainly consists of two types of lipophilic materials, cutin and cuticular wax. Cutin is the major structural component of the cuticle, which is composed of hydroxy and epoxy $\mathrm{C}_{16}$ and $\mathrm{C}_{18}$ fatty acid monomers, as well as glycerol (Nawrath, 2006). Cuticular waxes, as the second component of the cuticle, are either interspersed in the cutin matrix (cuticular waxes) or overlay the outermost surface of cutin polymer (epicuticular waxes). Cuticular and epicuticular waxes consist of complex mixtures of hydrophobic compounds, mostly very-long-chain fatty acids (VLCFAs) with more than 20 carbon atoms and their derivatives, including primary and secondary alcohols, aldehydes, alkanes, ketones and wax esters (Lemieux, 1996, Kunst and Samuels, 2003, Samuels et al., 2008). The cuticular wax composition and amounts vary greatly among plant species and tissues or organs, as well as development states (Lee and Suh, 2015). Primary alcohols and aldehydes are the major components of the epicuticular waxes in juvenile maize leaves (Javelle et al., 2010).

Epicuticular wax biosynthetic pathways have been extensively studied in Arabidopsis by identification and functional characterization of wax-deficient mutant genes (Lee and Suh, 2013). The first step in wax biosynthesis is the elongation of $\mathrm{C}_{16}$ and $\mathrm{C}_{18}$ fatty acids in the endoplasmic reticulum (ER) into VLCFAs by joining $\mathrm{C}_{2}$ building blocks of acetyl-coenzyme A into straight-chain of up to 34 carbon atoms via a fatty acid elongase (FAE) complex (Samuels et al., 2008, Kunst and Samuels, 2009). Following elongation, VLCFAs are modified into different wax products via the distinct alcohol-forming and the -forming pathways (Bernard and Joubes, 2013).

Secretion of epicuticular waxes was elucidated via the identification of two membrane-located ATP binding cassette (ABC) transporters, CER5 and WBC11, responsible for wax export across the PM (Pighin et al., 2004, Bird et al., 2007). However, the mechanism for intracellular trafficking of wax components from their site of synthesis at the ER to the PM is less clear. Through the characterization of the Arabidopsis ltpg mutant, lipid transfer proteins (LTPs) have been proposed to be involved in epicuticular wax deposition (Debono et al., 2009). In addition, deficient wax secretion in mutants of the Arabidopsis GNL1 or ECH genes that function in endomembrane vesicle trafficking indicated that both genes are involved in intracellular wax trafficking (McFarlane et al., 2014).

In maize (Zea mays), cuticular wax biosynthesis and accumulation have been aided by the identification of 
more than 30 glossy ( $g l$ ) loci (Schnable et al., 1994; and unpublished data from the Schnable lab), some of which have been cloned. $g l 1$ and $g l 2$, homologs of Arabidopsis CER3/WAX2 and CER2, are involved in leaf epicuticular wax alkane-forming pathway and the extension of VLCFAs to $\mathrm{C}_{30}$, respectively (Tacke et al., 1995, Negruk et al., 1996, Hansen et al., 1997, Sturaro et al., 2005). gl4 and gl8, homologs of KSC6 and KCR of Arabidopsis, belong to the fatty acid elongase (FAE) complex and play important roles in VLCFAs synthesis (Xu et al., 1997, Dietrich et al., 2005, Liu et al., 2009), respectively. gl3 and gl15 encode MYB and APETALA2 (AP2)-like transcription factors respectively, and both function in the regulation of epicuticular wax biosynthesis (Moose and Sisco, 1996, Liu et al., 2012). Recently, the gll3 gene was identified to encode a putative ABC transporter involved in the transport of epicuticular waxes (Li et al., 2013).

One of the most important functions of plant epicuticular waxes is to serve as a protective barrier against environmental stresses, including drought. Drought stress alters the composition and increases the content of epicuticular waxes in Arabidopsis, rice and wheat in some cases, and epicuticular wax content has been associated with drought tolerance (Aharoni et al., 2004, Kosma et al., 2009, Zhu and Xiong, 2013, Zhang et al., 2015). Some genes involved in biosynthesis and transport of cuticular wax have been proven in improving plant drought tolerance in Arabidopsis and several crops (Lee and Suh, 2015, Xue et al., 2017). However, the role of cuticular wax accumulation in drought tolerance in maize remains unclear.

Here, we report the cloning of the maize glossy6 ( $g l 6)$ gene that is involved in epicuticular wax accumulation and show that the gl6 mutant, relative to wild type, exhibits reduced epicuticular wax accumulation, as well as increased cuticle permeability and seedling drought sensitivity. Functional characterization indicated that the GL6 protein might be involved in intracellular transport of cuticular wax, providing novel insight into the epicuticular wax biosynthesis/transport pathway.

\section{RESULTS}

\section{Morphological and biochemical characterization of the gl6 mutant}

The spontaneous gl6 mutant first described by Emerson in 1935 has been designated gl6-ref (Emerson et al., 1935, Schnable et al., 1994). Like other glossy mutants in maize, seedling leaves of the gl6-ref mutant are shiny green in appearance, and water droplets easily form and adher to leaf surfaces after leaves are sprayed with water (Figure 1a). The accumulation of epicuticular waxes on the second leaf surface of gl6-ref mutants was examined via Field Emission Scanning Electron Microscopy (FE-SEM). Substantially fewer wax crystals were observed on 
surfaces of gl6-ref mutant leaves relative to surfaces of wild type leaves (Figure 1b). Ultrastructure analysis of leaf epidermal cells by transmission electron microscope (TEM) found acerose inclusions in epidermal cells of gl6-ref mutants (Figure 1d, e), but not in wild-type epidermal cells (Figure 1c). This finding is similar to the observation of linear inclusions in Arabidopsis cer 5 mutants which exhibit defects in export of waxes through the PM (Pighin et al., 2004). Our results, therefore, suggested that waxes have accumulated within $g l 6$ cells.

The wax load and composition of gl6-ref mutants were assessed via GC-mass spectrometry. Relative to wild type, leaf surface waxes loads on $\mathrm{gl} 6$ mutants were reduced by $80 \%$, from $13.2 \mu \mathrm{g} / \mathrm{cm}^{2}$ wild-type level to 2.6 $\mu \mathrm{g} / \mathrm{cm}^{2}$ (Figure 2a). Wax composition analysis showed that aldehydes and primary alcohols were highly reduced in gl6 mutants, which decreased to $1.5 \%$ and $22 \%$ of wild-type levels, respectively. In addition, the accumulations of fatty acids, alkanes, ethyl stearate, $\beta$-sitosterol, and several unidentified wax classes were also decreased in $g l 6$ mutants (Figure 2b). For individual wax constituents, amounts of $\mathrm{C}_{32}$ aldehydes and $\mathrm{C}_{32}$ primary alcohols on gl6 mutant leaves were significantly less than on wild-type leaves. Amounts of $\mathrm{C}_{18}, \mathrm{C}_{31}$ fatty acids, $\mathrm{C}_{29}, \mathrm{C}_{31}, \mathrm{C}_{32}$ alkanes, $\mathrm{C}_{30}, \mathrm{C}_{34}$ aldehydes and $\mathrm{C}_{30}, \mathrm{C}_{33}$ primary alcohols were also reduced in gl6 mutants (Figure $2 \mathrm{~b}$ ).

We further quantified total waxes including both surface and intracellular waxes and determined the proportion of total waxes secreted to the surface. Total waxes and the proportion of waxes secreted were determined according to published methods (McFarlane et al., 2014). As a result, total waxes were reduced from the wild type levels of $19.7 \mu \mathrm{g} / \mathrm{cm}^{2}$ to $13.1 \mu \mathrm{g} / \mathrm{cm}^{2}$ in the $\mathrm{gl} 6$ mutant (Table 1 ). In wild-type leaves, $67.1 \%$ of total waxes were from surface waxes, suggesting that most waxes were secreted (Table 1). In contrast, only $19.9 \%$ of total waxes were surface waxes in mutant leaves. In conjunction with the observation of acerose inclusions in gl6 epidermal cells (Figure 1d, e), these result suggest that the wax exporting system is dysfunctional, causing a large proportion of waxes to remain within cells. Taken together, these results indicated that $g l 6$ is involved in both the biosynthesis of very-long-chain waxes, particularly $\mathrm{C}_{32}$ aldehydes and $\mathrm{C}_{32}$ primary alcohols, and, possibly, the wax transport from the ER to the plant surface.

\section{Increased cuticle permeability and drought sensitivity of gl6 mutants}

Reduced epicuticular wax accumulation is associated with increased cuticle permeability (Kerstiens, 1996, Aharoni et al., 2004, Bessire et al., 2007). We performed chlorophyll leaching and water loss assays using seedling leaves of gl6-ref mutants and wild types. For the chlorophyll leaching assay, the concentration of leaf

chlorophyll in the solution was monitored at various time points. The results showed that leaf chlorophyll leaching of gl6-ref mutants was faster than that of wild type (Figure 3d), even though the total leaf chlorophyll 
contents of gl6-ref mutants and wild types were similar (Figure 3c). To detect water loss, detached leaves of gl6-ref and wild type were exposed to air at room temperature; gl6-ref mutant leaves were obviously curled and wilted after only 2 hours (Figure 3a). The rate of water loss of detached leaves from gl6-ref mutants was significantly higher than that of wild types (Figure 3b). Results from both the chlorophyll leaching and water loss assays suggested that gl6-ref mutant leaves exhibit increased cuticle permeability as compared with wild-type leaves.

Leaf water loss has been associated with reduced leaf surface temperature. Hence, monitoring leaf surface temperature is widely used as an indicator of leaf water loss (Mustilli et al., 2002). The surface temperature of gl6-ref mutant leaves, monitored via infrared thermography, was lower than that of wild-type leaves under both drought stress and well-watered conditions (Figure 4e), while stomata density, pavement cell density, and stomata index of gl6-ref mutants were similar to those of wild type (Figure 4f, g and h). These results suggested that the decreased leaf wax accumulation of $g$ l6-ref mutants caused faster water losses.

Given the increased cuticle permeability and leaf water loss of the gl6-ref mutant, we investigated how gl6-ref mutant seedlings respond to drought stress. As a result, gl6-ref mutants showed a more severe wilting phenotype as compared with wild-type controls (Figure 4a). After re-watering, about $50 \%$ of wild-type seedlings could recover and survive, whereas none of gl6-ref mutant seedling plants could survive (Figure 4a, b). Under the well-watered conditions, no significant phenotypic differences in either the wilting phenotype or the relative water content of leaves were observed between gl6-ref mutants and wild-type seedlings (Figure 4a, 4c). In contrast, under mild-drought stress, the relative water content of gl6-ref mutant seedling leaves was lower than that of wild-type controls. The re-watering plants after drought stress showed no significant differences in the leaf relative water content between two genotypes (Figure 4c). In addition, leaf electrolyte leakage assay for evaluating membrane damage was conducted, and the results showed that the gl6-ref mutant exhibited a higher relative electrical conductivity than that of wild type under mild-drought stress (Figure 4d), which indicated that a larger degree of membrane damage in mutants resulted in higher electrolyte leakage. In re-watered plants, no significant differences of the relative conductivity were observed between mutants and wild types (Figure 4d). The results corroborated that the gl6-ref mutation reduced seedling tolerance to drought.

\section{Molecular cloning of $g l 6$}

Previously, the gl6 gene had been mapped roughly to the long arm of chromosome 3 (Schnable et al., 1994). BSR-Seq, a method utilizing RNA-Seq for bulked segregant analysis (Liu et al., 2012) was performed to map the 
gene. Briefly, segregating $\mathrm{BC}_{8} \mathrm{~F}_{2}$ populations derived from the self-pollination of gl6-ref heterozygous plants were used to identify and collect mutant and wild-type plants, separately pooled into two bulks for RNA extraction and sequencing. Three replicates were conducted, producing six RNA-Seq data sets, each of which had more than 20 million reads. RNA-Seq reads were aligned to the B73 reference genome (AGPv2), and the polymorphic SNPs identified which were used as genetic markers to map the target gene. Through this process, the gl6 gene was mapped to an $18.9 \mathrm{Mb}$ interval on chromosome 3, from 113.5 to $132.4 \mathrm{Mb}$ (Figure $5 \mathrm{a}$ ).

To facilitate the identification of the $g l 6$ gene, additional $g l 6$ alleles were generated through $M u$ transposon mutagenesis and chemical ethyl methanesulfonate (EMS) treatment (Candela and Hake, 2008). Four new $M u$ tagged alleles were identified in the progeny of crosses between homozygous gl6-ref mutants and $M u$-active lines; these mutants were designated: $g l 6-2$, gl6-3, gl6-4 and gl6-5 (Table S1). Meanwhile, an EMS mutagenesis screen identified three glossy mutants that were verified to be allelic to gl6-ref, and which were termed gl6-6, gl6-7, and gl6-8 (Table S1).

One $M u$ tagged allele, gl6-2, was used to perform Seq-Walking sequencing, a genome walking approach to amplify and isolate DNA fragments flanking $M u$ transposon insertions throughout the genome (Li et al., 2013). In total, 28 non-redundant $M u$ flanking sequence (MFS) sites with >30 reads were identified in the $18.9 \mathrm{Mb}$ BSR-Seq mapping interval, and one $M u$-insertion hotspot gene, GRMZM2G139786 containing three MFS was identified (Figure S1, Table S2). At the same time, gl6-2 allele was also subjected to DLA analysis, an adaptor-mediated PCR-based method for isolating $M u$ flanking sequences that co-segregated with mutant phenotypes (Liu et al., 2009). The DLA result independently identified a $M u$ insertion in GRMZM2G139786 that co-segregated the $g l 6$ glossy phenotype (Figure 5b). Based on these results, all four $M u$-derived alleles, $g l 6-2$, gl6-3, gl6-4 and $g l 6-5$ were sequenced to identify mutations in GRMZM2G139786. Mul insertions were identified $37 \mathrm{bp}$ upstream of the $g l 6$ start codon in $g l 6-2,211$ bp upstream of the start codon in both $g l 6-4$ and gl6-5 alleles (Dietrich et al., 2002), and 320 bp downstream of the start codon in gl6-3 allele (Figure 5c).

The genomic sequences of GRMZM2G139786 from three EMS-induced gl6 alleles were amplified and Sanger sequenced (Table S1). These analyses showed that the gl6-6 allele contains a G to A transition $314 \mathrm{bp}$ downstream of the start codon, producing a premature termination codon (PTC); gl6-7 carries a C to T transition 391 bp downstream of the start codon, causing an amino acid change from arginine to cysteine; and the $g l 6-8$ allele contains a 9-bp deletion 497bp downstream of the start codon and seven non-synonymous mutations in the coding region (Table S1, Figure 5c). In addition, based on the BSR-Seq result, the accumulation of the GRMZM2G139786 transcripts was significantly reduced in the gl6-ref mutant pool as compared to the wild-type 
pool (Table S3). Collectively, these results demonstrate that GRMZM2G139786 is the gl6 gene.

\section{Characterization of the novel glossy gene, gl6}

The gl6 gene has a single exon with a 675 -bp open reading frame and encodes a putative protein with 224 amino acids. Domain analysis showed that GL6 is a novel protein containing a conserved DUF538 (domain of unknown function) between positions 29-138 aa of the protein (Figure S2). Homologs of GL6 can be identified in sorghum, rice, Arabidopsis and other plants. Phylogenetic analysis using the full-length protein sequences of GL6 homologs showed that GL6 was grouped into a monocot-specific subfamily (Figure 5d), and multiple sequence alignment showed that the DUF538 domain of these proteins is highly conserved (Figure S2).

A fusion construct of GL6 with C-terminal Yellow Fluorescent Protein (YFP) was generated and expressed in maize protoplast cells. Observation via confocal microscopy showed that GL6-YFP fluorescence signals were was detected in the cytoplasm and the plasma membrane, but not in the nucleus, whereas the control YFP signal was observed throughout the cell (Figures $6 \mathrm{a}, \mathrm{b}$ ). To further study the compartmental localization of the GL6 protein, we co-expressed GL6-YFP with the endoplasmic reticulum (ER) marker red fluorescent protein (RFP)-CNX, the Golgi marker mRFP-ManI, the trans-Golgi network (TGN) marker RFP-SYP41 and the nuclear protein marker mRFP-AHL22 in the protoplast cells, and the results revealed that GL6-YFP co-localized with the ER, Golgi and TGN marker (Figures 6c, d and e), but not with the nuclear protein marker (Figure S3). To confirm plasma membrane localization of GL6, the GL6-YFP expressing cells were incubated with the plasma membrane marker FM4-64, and the result showed that the signals of GL6-YFP and FM4-64 were co-localized as expected (Figure 6f). Together, our evidence indicated that GL6 is an ER membrane, Golgi, TGN and plasma membrane-localized protein.

Real-time quantitative RT-PCR analysis was performed to detect gl6 gene expression in different maize tissues, and the results showed that gl6 was significantly expressed in leaves and silk, especially in young leaf, but lower expressed in root, husk, anther and immature seed (Figure S4). To further understand the impact of the gl6 mutation on the transcriptome, we analyzed differential expression in gl6 mutants and wild types using the BSR-Seq data. With a $10 \%$ false discovery rate (FDR), 421 differential expression (DE) genes were identified (Table S4). Among the DE genes, 235 and 186 genes were up- and down-regulated in the gl6 mutant versus wild type, respectively (Figure 7a). Gene Ontology (GO) enrichment analysis was performed on these DE genes using AgriGO (Du et al., 2010), and complete lists of significantly enriched GO terms are shown in Table S4. Significant enrichment of cellular response to stimulus and stress, especially to water and heat stresses were observed among the enriched GO terms (Figure 7b). Moreover, an enrichment of GO terms related to fatty acid 
biosynthetic process was also observed. Of genes in these GO terms, two genes, GRMZM2G029912 and GRMZM2G083526, which are homologs of the Arabidopsis CER3/WAX2 gene (AT5G57800) involved in the biosynthesis of VLCFAs, and another gene, GRMZM2G031790, a homolog of the Arabidopsis KCS2 gene (AT1G04220), showed significantly higher expression in the $g l 6$ mutant with 12.0, 4.8 and 12.5 fold changes, respectively (Lee et al., 2009, Bernard et al., 2012) (Table S3). This result suggested that altering wax amount and localization in the gl6 mutant resulted in feedback, causing up-regulation of some genes in the fatty acid biosynthesis pathway.

\section{DISCUSSION}

In this study, multiple strategies were combined to facilitate the genetic mapping and the identification of a gl6 candidate gene, which was confirmed using multiple independent $M u$-tagged and EMS-induced $g l 6$ alleles.

The $g l 6$ is a novel gene with a conserved uncharacterized domain, opening a door to extending our understanding of the molecular basis of epicuticular wax accumulation.

The phenotype and physiological characterization of the gl6 mutant showed that $g l 6$ is a typical maize glossy mutant with reduced epicuticular wax accumulation on the leaf surface, resulting in a glossy phenotype. Epicuticular waxes, the hydrophobic layer on plant surfaces, protect leaves from non-stomatal water loss (Samuels et al., 2008). However, few studies provide quantification data to measure drought tolerance of maize glossy mutants. We demonstrated the reduction of epicuticular waxes in the gl6 mutant was associated with increased cuticle permeability and reduced drought tolerance of maize seedlings. Studies from other plant species have shown that increases in epicuticular wax load can enhance drought tolerance (Lu et al., 2012, Luo et al., 2013, Zhou et al., 2013, Zhu and Xiong, 2013, Al-Abdallat et al., 2014). However, epicuticular wax does not accumulate to high lvels on the leaves of adult maize plants. Future studies should test the impact of elevated expression of epicuticular wax on plant drought tolerance in adult plants as well as the potential physiological cost of accumulating abundant epicuticular waxes.

Sequence analysis revealed that $g l 6$ encodes a novel protein containing a conserved DUF538 domain with no defined function. The gl6 orthologs were identified in sorghum, rice, Arabidopsis and other plants, but none has been characterized. In this study, we determined that maize gl6 is involved in wax accumulation and drought tolerance, which will provide insights into understanding the molecular function of DUF538 family members in other plants. In Arabidopsis, a wax-deficient phenotype is associated with organ fusion including different 
abnormalities in trichome development (Wellesen et al., 2001, Chen et al., 2003, Kurata et al., 2003). A T-DNA insertion mutation of the gl6 homologous gene At1g56580, named svb-1, has been examined in Arabidopsis, finding smaller trichome with variable branches in $s v b-1$ mutants versus wild types (Marks et al., 2009). However, no phenotype associated with epicuticular wax accumulation in the $s v b-1$ mutant was reported, which is worth further investigation on the basis of our findings.

The gl6 gene encodes a novel protein. Our characterizations have provided clues as to its function. VLCFAs are synthesized in the ER but the processes by which wax components from the ER are delivered to the plasma membrane remain unknown. Two hypotheses have been proposed, Golgi-mediated vesicular trafficking pathway and transfers via physical ER-PM connections (Kunst and Samuels, 2003, Schulz and Frommer, 2004). Two vesicle-trafficking genes, GNLI and $E C H$, have been implicated in wax transport from the ER to the PM (McFarlane et al., 2014), which supports the Golgi-mediated vesicular trafficking hypothesis. In the current study, the GL6 protein was localized in the ER membrane, the Golgi, the trans-Golgi network (TGN), and the plasma membrane, which provides further support for the involvement of the Golgi in intracellular wax transport. Our results also suggested that GL6 might play a role in the process of extracellular wax transport. Quantification of secreted waxes on the leaf surface and total leaf waxes showed that a decrease of total waxes in the mutant largely attributed to the reduced accumulation of surface waxes. Ignoring surface waxes, the remaining wax accumulation in mutants was higher than that in wild types, indicative of dysfunctional wax export to leaf surfaces in $g l 6$ mutants. Acerose inclusions found in epidermal cells of the gl6 mutant via TEM analysis provided further evidence that waxes accumulate inside epidermal cells of gl6 mutants. Taken together, our results suggest that GL6 functions in the intracellular transport of cuticular waxes, thereby influencing epicuticular wax accumulation.

\section{EXPERIMENTAL PROCEDURES}

\section{Plant Materials}

The maize (Zea mays) glossy6 reference mutant allele (termed gl6-ref, Schnable lab Ac\#245) was obtained from in Maize Genetics Stock Center and maintained in the Schnable lab (Schnable et al., 1994). Four additional glossy6 alleles (gl6-2, gl6-3, gl6-4 and gl6-5) isolated via $M u$ transposon tagging screens in Schnable lab from 1992 to 2010 were used to clone the gl6 gene via Seq-Walking and DLA analysis. Three EMS-induced alleles ( $g$ l6-6 and gl6-7 were generated by the Schnable lab; gl6-8 was generated by M.G Neuffer's lab, GN) were also 
screened and used to verify the candidate gl6 gene in this study (Table S1; Schnable et al., 1994).

The gl6-ref mutant has been backcrossed to B73 inbred line background up to 8 generations and then continuously self-pollinated. $\mathrm{BC}_{8} \mathrm{~F}_{2}$ segregating populations from this backcrossing program were used to map the $g l 6$ gene by the BSR-Seq technology, and subsequent $\mathrm{BC}_{8} \mathrm{~F}_{3}$ homozygous gl6 mutant or wild type lines were used for the drought tolerance assay, physiology characterization and leaf surface wax analyses.

\section{Electron Microscopy Techniques}

The second leaves collected from gl6-ref mutant and wild type were used for FE-SEM analysis according to the standard protocols (Aharoni et al., 2004). In brief, samples were fixed on the spindle and frozen in liquid nitrogen, dried in a vacuum-drying oven and then characterized by FE-SEM microscope (SU8010, HITACHI, Japan).

The collected leaves were also used for TEM analysis by the conventional chemical protocols (Chen et al., 2003). Samples were fixed in $2 \%$ glutaraldehyde in $0.1 \mathrm{M}$ phosphate buffer (pH 7.4), post-fixed in $1 \%$ osmium tetroxide for $1 \mathrm{~h}$, dehydrated with gradient alcohol solutions, and embedded in LR White resin. Ultrathin cross-sections were prepared with a Leica EM UC6 ultramicrotome, stained with $2 \%$ uranyl acetate for $2 \mathrm{~h}$, and were analyzed with transmission electron microscope (H-7500, HITACHI, Japan).

To measure the stomatal and pavement cell density and calculate the stomatal index, imprints were detached from the surface of the collected leaves and were mounted on glass microscope (Nadakuduti et al., 2012). Samples were observed with an optical microscope (IX71, Olympus, Japan).

\section{Water Loss and Chlorophyll Leaching Assay}

Detached leaves of gl6-ref mutant and wild-types were left at room temperature and photographed and weighed every $1 \mathrm{~h}$. Water loss was calculated and represented by the percentage of fresh weight. Chlorophyll leaching assays were carried out according as described (Aharoni et al., 2004). Briefly, excised seedling leaves were washed with tap water, weighed, and put in $30 \mathrm{~mL}$ of $80 \%$ ethanol at room temperature. Four hundred microliters were taken out from each sample every $10 \mathrm{~min}$, and were used to measure the absorbance at 664 and $647 \mathrm{~nm}$ wavelength. Finally, the samples were incubated in boiling water, cooled on ice, and were used to examine the absorbance to evaluate the total chlorophyll content. The formula in the described method was used to calculate the chlorophyll content and chlorophyll extraction rate.

\section{Drought Tolerance Experiment}

Seeds of the $\mathrm{BC}_{8} \mathrm{~F}_{3}$ homozygous gl6 mutant or wild type were germinated in paper towels for 2 days and 
then transplanted in sand-filled pots in a greenhouse $\left(27^{\circ} \mathrm{C}\right.$ day $/ 23^{\circ} \mathrm{C}$ night, $16 \mathrm{~h}$ day $)$. The control plants were well watered, and the treatment plants were subjected to drought stress by by withholding water for up to 20 days, and re-watered. Six biological replications were used for this assay, and the number of surviving seedlings used to calculate the survival rate. During the drought treatment, the relative water content (RWC) and relative electrical conductivity (REC) were respectively monitored by the previously described method (Zheng et al., 2010).

Thermal images were obtained using an infrared imaging system (VarioCam HD, InfraTec, Germany) following the manufacturer's instructions.

\section{Analysis of Wax Composition}

Wax extraction, gas chromatography (GC)-mass spectrometry (MS) analyses were performed according to the described methods with some modifications (Chen et al., 2011). The gl6 mutants and the wild-types grown in the substrate of roseite and sands $(1: 1)$ at growth chamber $\left(25^{\circ} \mathrm{C}\right)$ under $16 / 8 \mathrm{hrs}$ light/dark for three-leaves (about 6-7 days after plant, DAP), the second leaves (6 DAP) contained 5-6 g dry matter from gl6 mutant and wild type were fresh collected and instantly immersing into 1,000 $\mu$ chloroform for $45 \mathrm{~s}$, the extracts containing $10 \mathrm{mg}$ of tetracosane (Fluka) as an internal standard, was transferred into opened reactive vials, dried with nitrogen gas (Pressure Blowing Concentrator; N-EVAP), and derivatized by adding $20 \quad \mu l$ of N,N-bis-trimethylsilyltrifluoroacetamide (Macherey-Nagel) and $20 \mu \mathrm{l}$ of pyridine and incubated for $40 \mathrm{~min}$ at $70^{\circ} \mathrm{C}$. These derivatized samples were then analyzed by GC-FID (Agilent, Technologies) and GC-MS (Agilent gas chromatograph coupled to an Agilent 5973N quadrupole mass selective detector).

\section{BSR-Seq, Seq-Walking and DLA Analysis}

About twenty glossy and non-glossy seedling segregants from the gl6-ref $\mathrm{BC}_{8} \mathrm{~F}_{2}$ population were collected to construct mutant and non-mutant bulks for RNA extraction. Total RNA was isolated using RNeasy Plus Mini Kit (Qiagen, USA) and RNA quality (RIN scores over 8) was checked on a Bioanalyzer 2100 (Agilent technologies, Life technology, USA) using an RNA 6000 Nano chip. RNA-Seq libraries were constructed using the Illumina RNA-Seq sample preparation kit according to the manufacturer's protocol. The RNA-Seq libraries were sequenced on an Illumina HiSeq2000 instrument. Sequencing data were used to perform BSR-Seq analysis following our published method (Liu et al., 2012). For the Seq-Walking analysis, DNA was extracted using a CTAB method (Murray and Thompson, 1980) from the pools of heterozygous of B73 $\times(\mathrm{gl6}-\mathrm{Mu} / \mathrm{gl6}-\mathrm{ref})$, in which half of the individuals contains $M u$-insertion allele, gl6-2 (Table S1). Following quantification, DNA samples were sheared using a Biorupter machine (Diagenode, USA) and sequenced. The resulting sequence data were 
analyzed according to our published Seq-Walking Strategy (Li et al., 2013). DNA samples of gl6-2 were also used to perform Digestion-Ligation-Amplification (DLA) analysis according to our published method (Liu et al., 2009).

\section{Transcriptome analysis}

RNA-Seq data of the gl6 mutant and non-mutant bulks used in the BSR-Seq analysis were also used to compare transcriptome-wide differences between the mutant and its wild-type control. Reads were aligned to the maize reference genome B73 AGPv3 using Tophat2 (Kim et al., 2013). Transcript accumulation levels were calculated using the R package HTSeq (Anders et al., 2015) and differentially expressed genes were identified with the R package DESeq2 (Love et al., 2014). Tests for enrichment of differentially expressed genes were performed using agroGO with the standard settings (Fisher's exact test with significance threshold of 0.05 , Yekutieli multi-test adjustment) (Du et al., 2010).

\section{qRT-PCR}

For tissue-specific expression analysis, samples of young roots and leaves of 12 and 24 days after sowing (DAS), mature leaves of 12 and 27 days after pollination (DAP), husk, silk, anther, endosperm, embryo and seeds of 14 and 27 DAP were collected from the inbred line B73, and then were used to extract total RNAs by TRIzol reagent (Invitrogen). Quantitative real-time RT-PCR (qRT-PCR) analysis was conducted with TransStart Green qPCR SuperMix (TransGen Biotech) on a 7300-sequence detection system (Applied Biosystems). Maize actin1 was used as internal control, and the relative expression of $g l 6$ mRNA was calculated using the $2^{-\Delta \Lambda C t}$ method (Livak and Schmittgen, 2001).

\section{Subcellular Location of GL6}

To generate the GL6-YFP fusion construct, the open reading frame (ORF) of the Gl6 gene was cloned into the pEarleyGate-101 vector which contains the yellow fluorescent protein (YFP) reporter gene (Earley et al., 2006). Using previously described PEG-mediated transformation protocols (Chen et al., 2014), the GL6-YFP construct was transformed into maize protoplasts alone or co-transformed with a ER marker (red fluorescent protein (RFP)-CNX), a Golgi marker (mRFP-ManI), a trans-Golgi network (TGN) marker (RFP-SYP41), or and a nuclear protein marker (mRFP-AHL22) (Xiao et al., 2009, Cui et al., 2014). Fluorescence signals were observed under a confocal laser scanning system (Leica Microsystems, Wetzlar, Germany).

\section{ACKNOWLEDGEMENTS}

This work was supported by the National Key Research and Development Program of China (Grant No. 
2016YFD0101002), the 948 project of the Ministry of Agriculture of China (Grant No. 2015-Z11), the Agricultural Science and Technology Innovation Program of CAAS and the National Natural Science Foundation of China (31701437). We thank Mr. Cheng-Ting "Eddy" Yeh, Drs. Wei Wu, Heng-Cheng Hu and An-Ping Hsia for technical support and helpful discussions, Ms. Mitzi Wilkening for sequencing services in ISU Genomic Technologies Facility, former members of the Schnable lab including the late Joel Hansen and Philip Stinard, and the Schnable Lab's current nursery manager, Ms. Lisa Coffey for the generation and maintenance of genetic stocks used in this study. We thank Dr. Jianxin Shi, Dr. Guorun Qu, and Ms. Qian Luo from Shanghai Jiao Tong University for their assistance in conducting the GC-FID and GC-MS measurements and associated data analyses.

\section{CONFLICT OF INTEREST}

The authors declare no conflict of interest.

\section{SUPPORTING INFORMATION}

Figure S1. Mu insertion sites from Seq-walking.

Figure S2. Multiple sequence alignment of amino acid sequences of the predicted maize GL6 protein and its homologs in other species.

Figure S3. Co-localization of GL6-YFP with mRFP-AHL22 (nuclear marker).

Figure S4. Accumulation of g16 transcripts in various tissues.

Table S1. gl6 alleles information and the corresponding mutation types

Table S2. $M u$ flanking sequences and $M u$ sequences for each $g l 6 M u$ tagging allele

Table S3. Differential expressed genes (DEGs) in g16 mutant vs. wild-types

Table S4. Enriched GO terms in gl6 mutant differentially expressed genes 


\section{REFERENCES}

Aharoni, A., Dixit, S., Jetter, R., Thoenes, E., van Arkel, G. and Pereira, A. (2004) The SHINE clade of AP2 domain transcription factors activates wax biosynthesis, alters cuticle properties, and confers drought tolerance when overexpressed in Arabidopsis. The Plant cell, 16, 2463-2480.

Al-Abdallat, A.M., Al-Debei, H.S., Ayad, J.Y. and Hasan, S. (2014) Over-expression of SiSHN1 gene improves drought tolerance by increasing cuticular wax accumulation in tomato. International journal of molecular sciences, 15, 19499-19515.

Anders, S., Pyl, P.T. and Huber, W. (2015) HTSeq--a Python framework to work with high-throughput sequencing data. Bioinformatics, 31, 166-169.

Bernard, A., Domergue, F., Pascal, S., Jetter, R., Renne, C., Faure, J.D., Haslam, R.P., Napier, J.A., Lessire, R. and Joubes, J. (2012) Reconstitution of plant alkane biosynthesis in yeast demonstrates that Arabidopsis ECERIFERUM1 and ECERIFERUM3 are core components of a very-long-chain alkane synthesis complex. The Plant cell, 24, 3106-3118.

Bernard, A. and Joubes, J. (2013) Arabidopsis cuticular waxes: advances in synthesis, export and regulation. Progress in lipid research, 52, 110-129.

Bessire, M., Chassot, C., Jacquat, A.C., Humphry, M., Borel, S., Petetot, J.M., Metraux, J.P. and Nawrath, C. (2007) A permeable cuticle in Arabidopsis leads to a strong resistance to Botrytis cinerea. The EMBO journal, 26, 2158-2168.

Bird, D., Beisson, F., Brigham, A., Shin, J., Greer, S., Jetter, R., Kunst, L., Wu, X., Yephremov, A. and Samuels, L. (2007) Characterization of Arabidopsis ABCG11/WBC11, an ATP binding cassette $(\mathrm{ABC})$ transporter that is required for cuticular lipid secretion. Plant J, 52, 485-498.

Candela, H. and Hake, S. (2008) The art and design of genetic screens: maize. Nature reviews, 9 , 192-203.

Chen, W., Yu, X.H., Zhang, K., Shi, J., De Oliveira, S., Schreiber, L., Shanklin, J. and Zhang, D. (2011) Male Sterile2 encodes a plastid-localized fatty acyl carrier protein reductase required for pollen exine development in Arabidopsis. Plant physiology, 157, 842-853.

Chen, X., Goodwin, S.M., Boroff, V.L., Liu, X. and Jenks, M.A. (2003) Cloning and characterization of the WAX2 gene of Arabidopsis involved in cuticle membrane and wax production. The Plant cell, 15, 1170-1185.

Chen, X., Huang, Q., Zhang, F., Wang, B., Wang, J. and Zheng, J. (2014) ZmCIPK21, a maize CBL-interacting kinase, enhances salt stress tolerance in Arabidopsis thaliana. International journal of molecular sciences, 15, 14819-14834.

Cui, Y., Zhao, Q., Gao, C., Ding, Y., Zeng, Y., Ueda, T., Nakano, A. and Jiang, L. (2014) Activation of the Rab7 GTPase by the MON1-CCZ1 Complex Is Essential for PVC-to-Vacuole Trafficking and Plant Growth in Arabidopsis. The Plant cell, 26, 2080-2097.

Debono, A., Yeats, T.H., Rose, J.K., Bird, D., Jetter, R., Kunst, L. and Samuels, L. (2009) Arabidopsis LTPG is a glycosylphosphatidylinositol-anchored lipid transfer protein required for export of lipids to the plant surface. The Plant cell, 21, 1230-1238.

Dietrich, C.R., Cui, F., Packila, M.L., Li, J., Ashlock, D.A., Nikolau, B.J. and Schnable, P.S. (2002) Maize $\mathrm{Mu}$ transposons are targeted to the 5' untranslated region of the gl8 gene and sequences flanking Mu target-site duplications exhibit nonrandom nucleotide composition throughout the genome. Genetics, 160, 697-716. 
Dietrich, C.R., Perera, M.A., M, D.Y.-N., Meeley, R.B., Nikolau, B.J. and Schnable, P.S. (2005) Characterization of two GL8 paralogs reveals that the 3-ketoacyl reductase component of fatty acid elongase is essential for maize (Zea mays L.) development. Plant J, 42, 844-861.

Du, Z., Zhou, X., Ling, Y., Zhang, Z. and Su, Z. (2010) agriGO: a GO analysis toolkit for the agricultural community. Nucleic acids research, 38, W64-70.

Earley, K.W., Haag, J.R., Pontes, O., Opper, K., Juehne, T., Song, K. and Pikaard, C.S. (2006) Gateway-compatible vectors for plant functional genomics and proteomics. Plant J, 45, 616-629.

Emerson, R. A., Beadle, G. W. and Fraser,. A. C. (1935) A summary of linkage studies in maize. Memoir, 180. Cornell university agricultural experiment station. Ithaca, New York: Published by the university.

Hansen, J.D., Pyee, J., Xia, Y., Wen, T.J., Robertson, D.S., Kolattukudy, P.E., Nikolau, B.J. and Schnable, P.S. (1997) The glossy1 locus of maize and an epidermis-specific cDNA from Kleinia odora define a class of receptor-like proteins required for the normal accumulation of cuticular waxes. Plant physiology, 113, 1091-1100.

Javelle, M., Vernoud, V., Depege-Fargeix, N., Arnould, C., Oursel, D., Domergue, F., Sarda, X. and Rogowsky, P.M. (2010) Overexpression of the epidermis-specific homeodomain-leucine zipper IV transcription factor Outer Cell Layer1 in maize identifies target genes involved in lipid metabolism and cuticle biosynthesis. Plant physiology, 154, 273-286.

Kerstiens, G. (1996) Cuticular water permeability and its physiological significance. Journal of experimental botany, 47, 1813-1832.

Kim, D., Pertea, G., Trapnell, C., Pimentel, H., Kelley, R. and Salzberg, S.L. (2013) TopHat2: accurate alignment of transcriptomes in the presence of insertions, deletions and gene fusions. Genome Biol, 14, R36.

Kosma, D.K., Bourdenx, B., Bernard, A., Parsons, E.P., Lu, S., Joubes, J. and Jenks, M.A. (2009) The impact of water deficiency on leaf cuticle lipids of Arabidopsis. Plant physiology, 151, 1918-1929.

Kunst, L. and Samuels, A.L. (2003) Biosynthesis and secretion of plant cuticular wax. Progress in lipid research, 42, 51-80.

Kunst, L. and Samuels, L. (2009) Plant cuticles shine: advances in wax biosynthesis and export. Current opinion in plant biology, 12, 721-727.

Kurata, T., Kawabata-Awai, C., Sakuradani, E., Shimizu, S., Okada, K. and Wada, T. (2003) The YORE-YORE gene regulates multiple aspects of epidermal cell differentiation in Arabidopsis. Plant J, 36, 55-66.

Lee, S.B., Jung, S.J., Go, Y.S., Kim, H.U., Kim, J.K., Cho, H.J., Park, O.K. and Suh, M.C. (2009) Two Arabidopsis 3-ketoacyl CoA synthase genes, KCS20 and KCS2/DAISY, are functionally redundant in cuticular wax and root suberin biosynthesis, but differentially controlled by osmotic stress. Plant J, 60, 462-475.

Lee, S.B. and Suh, M.C. (2013) Recent advances in cuticular wax biosynthesis and its regulation in Arabidopsis. Molecular plant, 6, 246-249.

Lee, S.B. and Suh, M.C. (2015) Advances in the understanding of cuticular waxes in Arabidopsis thaliana and crop species. Plant cell reports, 34, 557-572.

Lemieux, B. (1996) Molecular genetics of epicuticular wax biosynthesis. Trends in Plant Science, 1, 312-318. 
Li, L., Li, D., Liu, S., Ma, X., Dietrich, C.R., Hu, H.C., Zhang, G., Liu, Z., Zheng, J., Wang, G. and Schnable, P.S. (2013) The Maize glossy13 Gene, Cloned via BSR-Seq and Seq-Walking Encodes a Putative ABC Transporter Required for the Normal Accumulation of Epicuticular Waxes. PloS one, 8, e82333.

Liu, S., Dietrich, C.R. and Schnable, P.S. (2009) DLA-based strategies for cloning insertion mutants: cloning the g14 locus of maize using Mu transposon tagged alleles. Genetics, 183, 1215-1225.

Liu, S., Yeh, C.T., Tang, H.M., Nettleton, D. and Schnable, P.S. (2012) Gene Mapping via Bulked Segregant RNA-Seq (BSR-Seq). PloS one, 7, e36406.

Livak, K.J. and Schmittgen, T.D. (2001) Analysis of relative gene expression data using real-time quantitative PCR and the 2(-Delta Delta C(T)) Method. Methods, 25, 402-408.

Love, M.I., Huber, W. and Anders, S. (2014) Moderated estimation of fold change and dispersion for RNA-seq data with DESeq2. Genome Biol, 15, 550.

Lu, S., Zhao, H., Des Marais, D.L., Parsons, E.P., Wen, X., Xu, X., Bangarusamy, D.K., Wang, G., Rowland, O., Juenger, T., Bressan, R.A. and Jenks, M.A. (2012) Arabidopsis ECERIFERUM9 involvement in cuticle formation and maintenance of plant water status. Plant physiology, 159, 930-944.

Luo, X., Bai, X., Sun, X., Zhu, D., Liu, B., Ji, W., Cai, H., Cao, L., Wu, J., Hu, M., Liu, X., Tang, L. and Zhu, Y. (2013) Expression of wild soybean WRKY20 in Arabidopsis enhances drought tolerance and regulates ABA signalling. Journal of experimental botany, 64, 2155-2169.

Marks, M.D., Wenger, J.P., Gilding, E., Jilk, R. and Dixon, R.A. (2009) Transcriptome analysis of Arabidopsis wild-type and g13-sst sim trichomes identifies four additional genes required for trichome development. Molecular plant, 2, 803-822.

McFarlane, H.E., Watanabe, Y., Yang, W., Huang, Y., Ohlrogge, J. and Samuels, A.L. (2014) Golgi- and trans-Golgi network-mediated vesicle trafficking is required for wax secretion from epidermal cells. Plant physiology, 164, 1250-1260.

Moose, S.P. and Sisco, P.H. (1996) Glossy15, an APETALA2-like gene from maize that regulates leaf epidermal cell identity. Genes \& development, 10, 3018-3027.

Murray, M.G. and Thompson, W.F. (1980) Rapid isolation of high molecular weight plant DNA. Nucleic acids research, 8, 4321-4325.

Mustilli, A.C., Merlot, S., Vavasseur, A., Fenzi, F. and Giraudat, J. (2002) Arabidopsis OST1 protein kinase mediates the regulation of stomatal aperture by abscisic acid and acts upstream of reactive oxygen species production. The Plant cell, 14, 3089-3099.

Nadakuduti, S.S., Pollard, M., Kosma, D.K., Allen, C., Jr., Ohlrogge, J.B. and Barry, C.S. (2012) Pleiotropic phenotypes of the sticky peel mutant provide new insight into the role of CUTIN DEFICIENT2 in epidermal cell function in tomato. Plant physiology, 159, 945-960.

Nawrath, C. (2006) Unraveling the complex network of cuticular structure and function. Current opinion in plant biology, 9, 281-287.

Negruk, V., Yang, P., Subramanian, M., McNevin, J.P. and Lemieux, B. (1996) Molecular cloning and characterization of the CER2 gene of Arabidopsis thaliana. Plant J, 9, 137-145.

Pighin, J.A., Zheng, H., Balakshin, L.J., Goodman, I.P., Western, T.L., Jetter, R., Kunst, L. and Samuels, A.L. (2004) Plant cuticular lipid export requires an ABC transporter. Science (New York, N.Y, 306, 702-704.

Samuels, L., Kunst, L. and Jetter, R. (2008) Sealing plant surfaces: cuticular wax formation by epidermal cells. Annual review of plant biology, 59, 683-707. 
Schnable PS, Stinard PS, Wen TJ, Heinen S, Weber D, Zhang L, Hansen JD, Nikolau BJ (1994)

The genetics of cuticular wax biosynthesis. Maydica (Robertson issue), 39: 279-287.

Schulz, B. and Frommer, W.B. (2004) A plant ABC transporter takes the lotus seat. Science (New York, $N . Y$, 306, 622-625.

Shepherd, T. and Wynne Griffiths, D. (2006) The effects of stress on plant cuticular waxes. The New phytologist, 171, 469-499.

Sturaro, M., Hartings, H., Schmelzer, E., Velasco, R., Salamini, F. and Motto, M. (2005) Cloning and characterization of GLOSSY1, a maize gene involved in cuticle membrane and wax production. Plant physiology, 138, 478-489.

Tacke, E., Korfhage, C., Michel, D., Maddaloni, M., Motto, M., Lanzini, S., Salamini, F. and Doring, H.P. (1995) Transposon tagging of the maize Glossy2 locus with the transposable element En/Spm. Plant J, 8, 907-917.

Wellesen, K., Durst, F., Pinot, F., Benveniste, I., Nettesheim, K., Wisman, E., Steiner-Lange, S., Saedler, H. and Yephremov, A. (2001) Functional analysis of the LACERATA gene of Arabidopsis provides evidence for different roles of fatty acid omega -hydroxylation in development. Proceedings of the National Academy of Sciences of the United States of America, 98, 9694-9699.

Xiao, C., Chen, F., Yu, X., Lin, C. and Fu, Y.F. (2009) Over-expression of an AT-hook gene, AHL22, delays flowering and inhibits the elongation of the hypocotyl in Arabidopsis thaliana. Plant molecular biology, 71, 39-50.

Xu, X., Dietrich, C.R., Delledonne, M., Xia, Y., Wen, T.J., Robertson, D.S., Nikolau, B.J. and Schnable, P.S. (1997) Sequence analysis of the cloned glossy8 gene of maize suggests that it may code for a beta-ketoacyl reductase required for the biosynthesis of cuticular waxes. Plant physiology, 115, 501-510.

Xue, D., Zhang, X., Lu, X., Chen, G. and Chen, Z.H. (2017) Molecular and Evolutionary Mechanisms of Cuticular Wax for Plant Drought Tolerance. Frontiers in plant science, 8, 621.

Zhang, Z., Wei, W., Zhu, H., Challa, G.S., Bi, C., Trick, H.N. and Li, W. (2015) W3 Is a New Wax Locus That Is Essential for Biosynthesis of beta-Diketone, Development of Glaucousness, and Reduction of Cuticle Permeability in Common Wheat. PloS one, 10, e0140524.

Zheng, J., Fu, J., Gou, M., Huai, J., Liu, Y., Jian, M., Huang, Q., Guo, X., Dong, Z., Wang, H. and Wang, G. (2010) Genome-wide transcriptome analysis of two maize inbred lines under drought stress. Plant molecular biology, 72, 407-421.

Zhou, L., Ni, E., Yang, J., Zhou, H., Liang, H., Li, J., Jiang, D., Wang, Z., Liu, Z. and Zhuang, C. (2013) Rice OsGL1-6 is involved in leaf cuticular wax accumulation and drought resistance. PloS one, 8, e65139.

Zhu, X. and Xiong, L. (2013) Putative megaenzyme DWA1 plays essential roles in drought resistance by regulating stress-induced wax deposition in rice. Proceedings of the National Academy of Sciences of the United States of America, 110, 17790-17795. 
bioRxiv preprint doi: https://doi.org/10.1101/378687; this version posted July 27,2018 . The copyright holder for this preprint (which was not certified by peer review) is the author/funder. All rights reserved. No reuse allowed without permission.

\section{TABLES}

Table 1. Epicuticular wax extracted from the leaf surface compared with total wax of wild type and $g l 6$ mutant. $^{\mathrm{a}}$

\begin{tabular}{lcc}
\hline Parameter & WT & gl6-ref \\
\hline Total wax & $19.7 \pm 4.1$ & $13.1 \pm 2.4$ \\
Surface wax & $13.2 \pm 2.3$ & $2.6 \pm 0.5$ \\
Wax secreted ratio & $67.1 \%$ & $19.9 \%$ \\
\hline aTotal waxes extraction and & & \\
\hline
\end{tabular}

${ }^{a}$ Total waxes extraction and ratio of wax secretion calculation were performed following a reported measurement strategy (McFarlane et al., 2014). Total and surface wax content are means \pm SD $\left(\mathrm{ug} / \mathrm{cm}^{2}\right)$ 


\section{FIGURE LEGENDS}

Figure 1. Morphological characterization of the gl6 mutant. (a) gl6-ref mutant exhibits a glossy phenotype. Water is sprayed on seedlings to distinguish gl6 mutant seedlings from wild type (WT). (b) Leaf epicuticular wax accumulation in WT and mutant (gl6-ref) seedlings detected via SEM. 5000×magnification. (c) to (e), TEM analysis of leaf epidermal wax secreting cells wild type (WT) and gl6-ref mutant. (e) Enlarged view showing the red rectangle marked area in (d). Arrows in (d) and (e) indicate unusual linear inclusions. Bars $=3 \mathrm{~cm}$ in (a), $5 \mu \mathrm{m}$ in (b), (c) and (d), $500 \mathrm{~nm}$ in (e).

Figure 2. Total leaf epicuticular wax load and wax composition of wild type and gl6-ref mutant. (a) Total leaf cuticular wax load of wild type and gl6-ref mutant. (b) Epicuticular wax composition of wild type and gl6-ref mutant. Values are means of eight biological replicates \pm SD. UI indicates unidentified and asterisks indicate statistically significant differences between wild type and gl6 mutant $(* \mathrm{P}<0.05$, **P $<0.01, * * * \mathrm{P}<0.001$, Student's t test).

Figure 3. Leaf surface permeability analysis of gl6 mutant. (a) Detached leaves from WT and gl6-ref mutant immediately after harvest and after 2 hours at room temperature. (b) Water loss of detached leaves of the WT and gl6-ref mutant. (c) The total leaf chlorophyll content of WT and gl6-ref mutant. (d) Kinetics of chlorophyll leaching from leaves of the WT and gl6-ref mutant.

Figure 4. The gl6 mutant seedlings is sensitive to drought stress. (a) Drought phenotypes of WT and gl6-ref mutant seedlings in soil following drought stress and after re-watering. (b) The survival rate of WT and gl6 mutant seedings after drought stress and re-watering. (c) and (d), The leaf relative water content and relative electrical conductivity of WT and gl6 mutant seedlings under well watered (WW), drought stressed (DS) and re-watered (RW) conditions. In (b), (c) and (d), data are means of three replicates $\pm \mathrm{SD}(* * * \mathrm{P}<0.001$, Student's t test). (e) False-color 
infrared image of the wild type and the gl6 mutant under well watered and drought stressed conditions. (f) to (h), Stomatal density (f), pavement cell density (g), and stomatal index (number of stomata per total epidermal cells; $h$ ) analyzed in leaf abaxial epidermal layers from WT and g16 mutant. Data are means of five individual plants. Bars $=10 \mathrm{~cm}$ in (a) and (e).

Figure 5. Molecular cloning of the gl6 gene and its phylogenetic analysis. (a) BSR-Seq analysis of an $\mathrm{F}_{2}$ segregated gl6-ref population mapped gl6 to the 113.5-132.4 Mb interval of chr3. (b) Polyacrylamide gel electrophoresis (PAGE) results of Digestion-ligation-amplification (DLA) analysis using the adaptor primer (Nsp-15ctc). The red rectangle indicated the specific bands produced from g16/+ plants but not in WT plants. (c) The gene structure of $g l 6, M u$ insertions in four alleles and lesions in two gl6 EMS alleles. (d) Phylogenetic tree constructed using MEGA 7.0 and the GL6 protein and GL6 homologs aligned using ClustalW. Distances were estimated with a neighbor-joining algorithm, and bootstrap support is indicated to the left of branches.

Figure 6. Subcellular localization of the GL6 protein in maize protoplasts. Confocal images show the expression of the YFP, GL6 protein fused at its C terminus with YFP and organelle markers. (a) and (b) Subcellular localization YFP (a) and GL6-YFP (b). (c) Co-localization of GL6-YFP with RFP-CNX (an ER marker). (d) Co-localization of GL6-YFP with mRFP-ManI (a Golgi marker). (e) Co-localization of GL6-YFP with RFP-SYP41(a TGN marker). (f) Co-localization of GL6-YFP with FM4-64(a plasma membrane marker). Bar $=10 \mu \mathrm{m}$.

Figure 7. Summary of DE genes and GO Analysis. (a) Summary of the 421 genes that are differentially expressed between $g l 6$ and WT. The red and blue slices of the pie represent DE genes up- and down-regulated in the gl6 mutant, respectively. The different shades designate fold changes. (b) GO enrichment analysis of the $421 \mathrm{DE}$ genes. The GO analysis was performed using AgriGO (Du et al., 2010). The 
processes with deeper color exhibit more significant enrichment. The solid arrows means the process is the only downstream process of the upstream process, while dotted arrows means the process is one of multiple the downstream processes.

Figure S1. Mu insertion sites from Seq-walking.

Number of reads at each $M u$ insertion site is plotted versus site's physical coordinates.

Red dots highlight $M u$ insertion sites within the gl6 candidate gene. The region shown covers the mapping interval on chromosome 3.

Figure S2. Multiple sequence alignment of amino acid sequences of the predicted maize GL6 protein and its homologs in other species. Sequences were obtained from NCBI and aligned using the website

(http://multalin.toulouse.inra.fr/multalin/multalin.html) following default parameters. Zm, Zea mays; Do, Dichanthelium oligosanthes; Si, Setaria italic; $\mathrm{Ph}$, Panicum hallii; Sb, Sorghum bicolor; At, Aegilops tauschii; Bd, Brachypodium distachyon; Ob, Oryza brachyantha; Os, Oryza sativa; Tu, Triticum urartu.

Figure S3. Co-localization of GL6-YFP with mRFP-AHL22 (nuclear marker). Confocal images show the expression of GL6 protein fused at its C terminus with YFP and the nuclear marker mRFP-AHL22. Bar $=10 \mu \mathrm{m}$.

Figure S4. Accumulation of $g l 6$ transcripts in various tissues. Real-time quantitative RT-PCR analysis of roots, leaves, husk, silk, anther and seeds from the inbred line B73, normalized using the maize tublin gene. Values are means of three replicates \pm SD. DAS indicates days after sowing, DAP indicates days after pollination. 
(a)

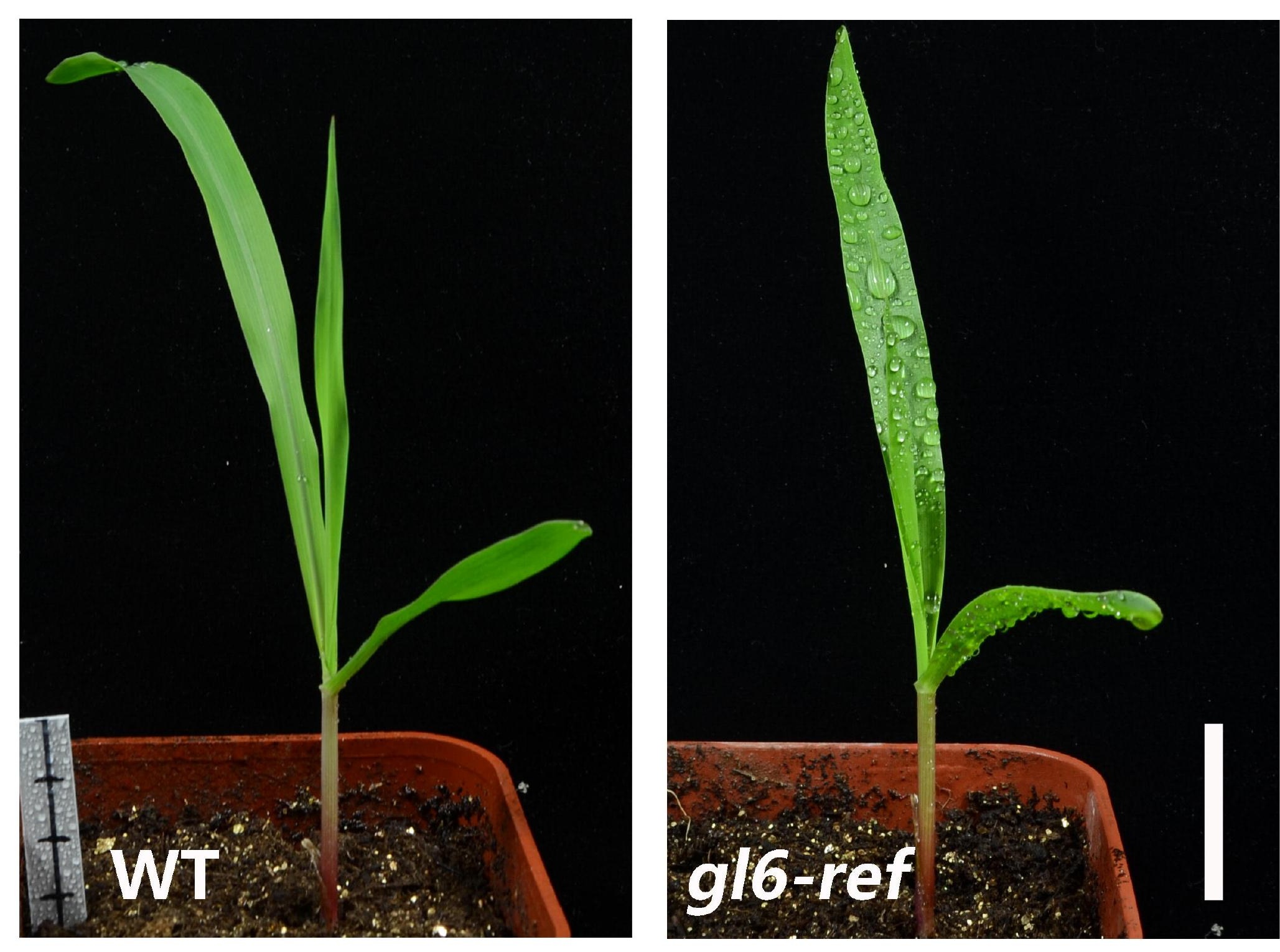

(b) WT

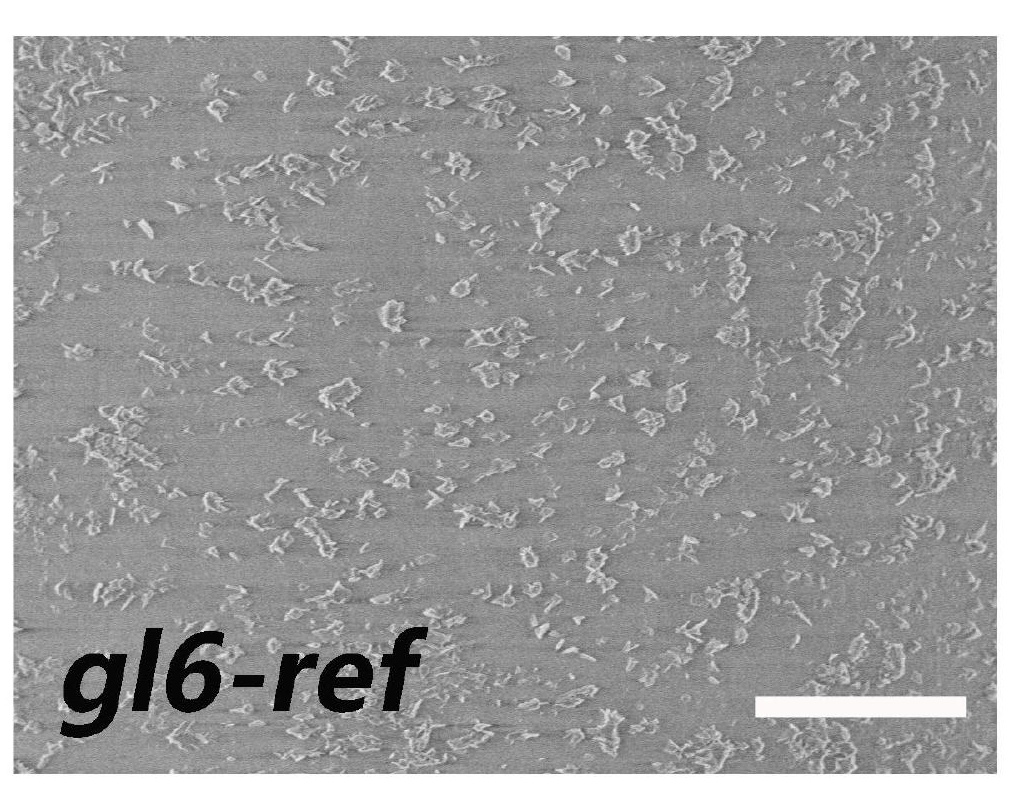

(c)

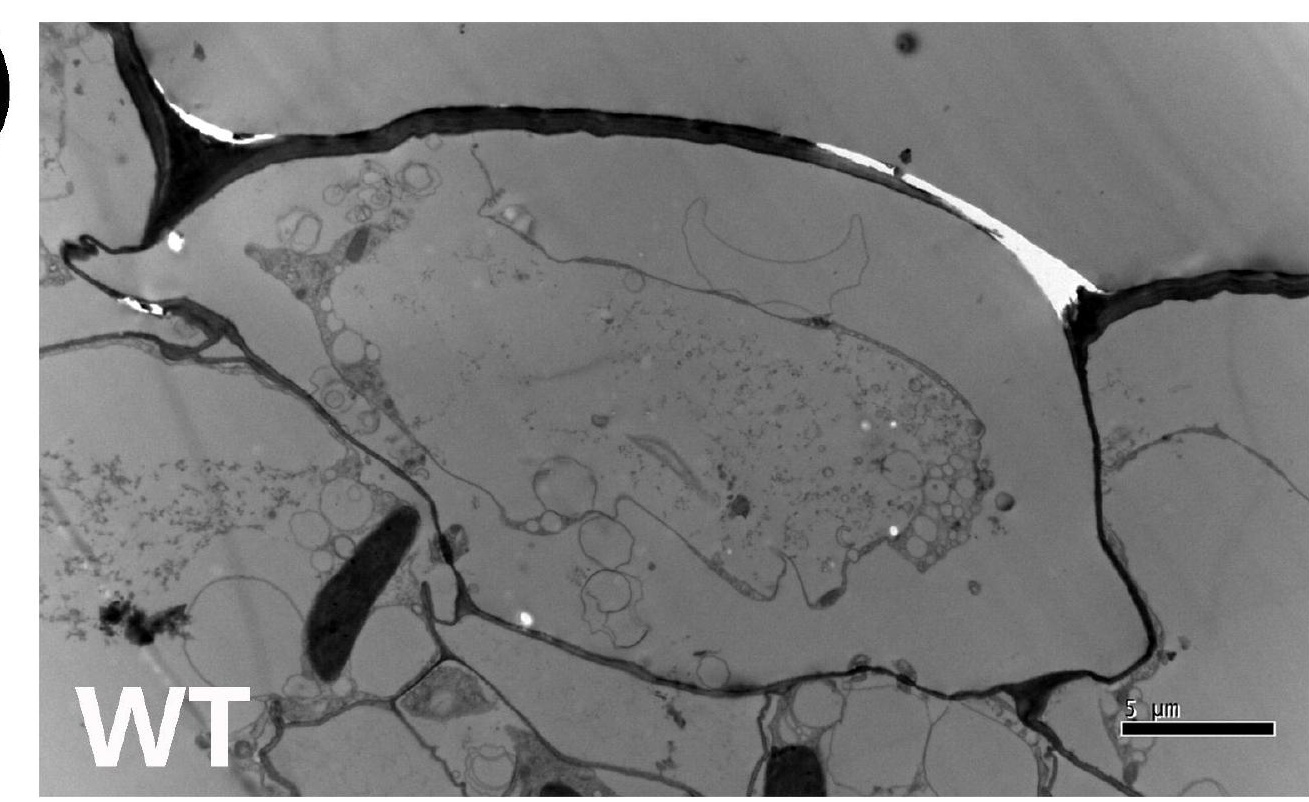

(d)

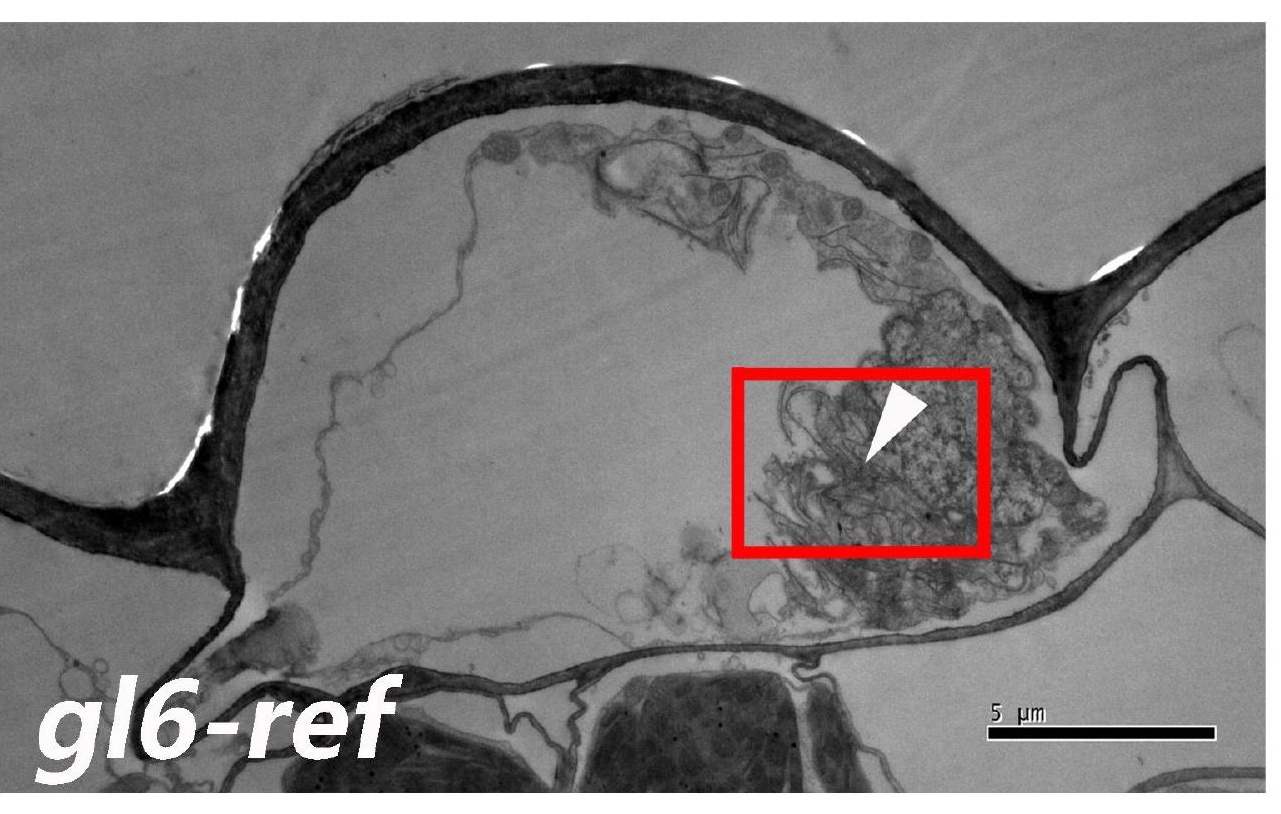

(e) g) $6 \mathrm{ref}$ 
(a)

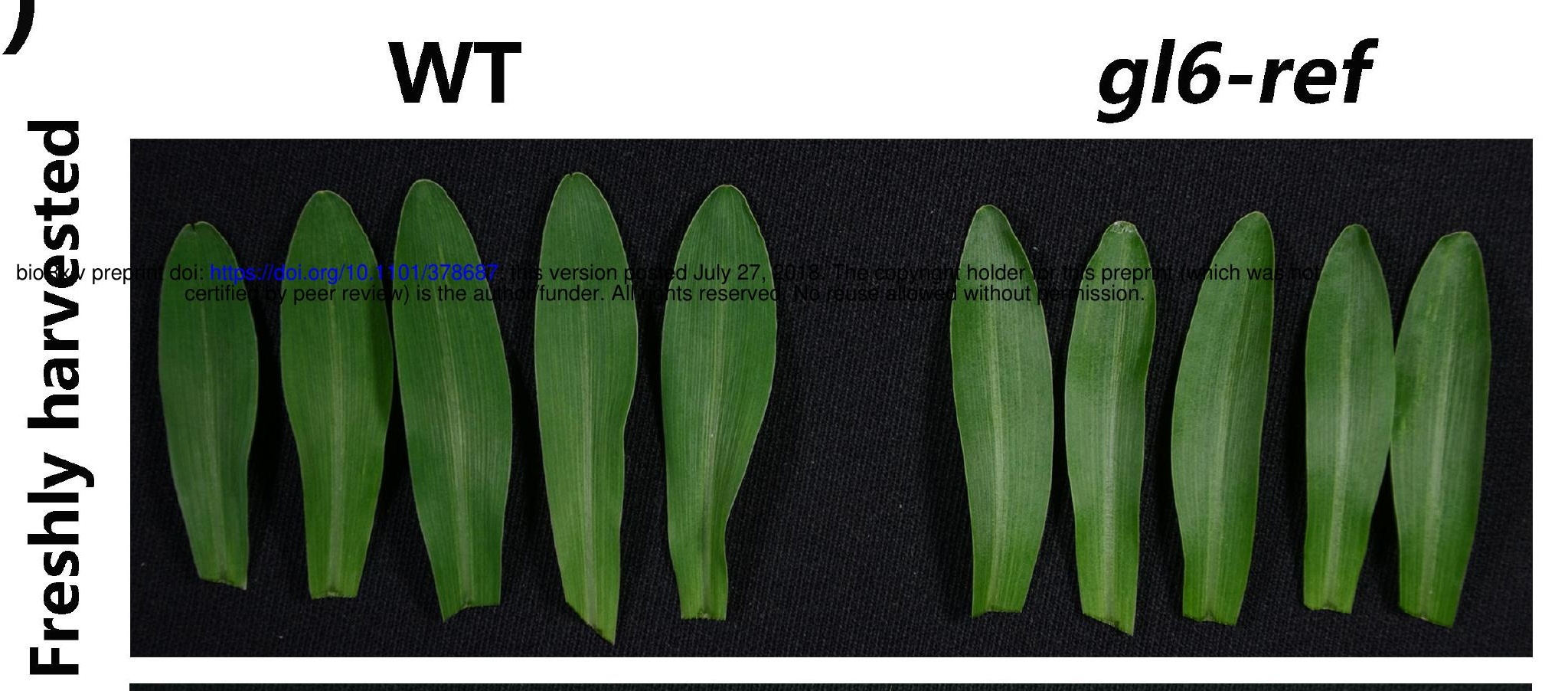

(c)

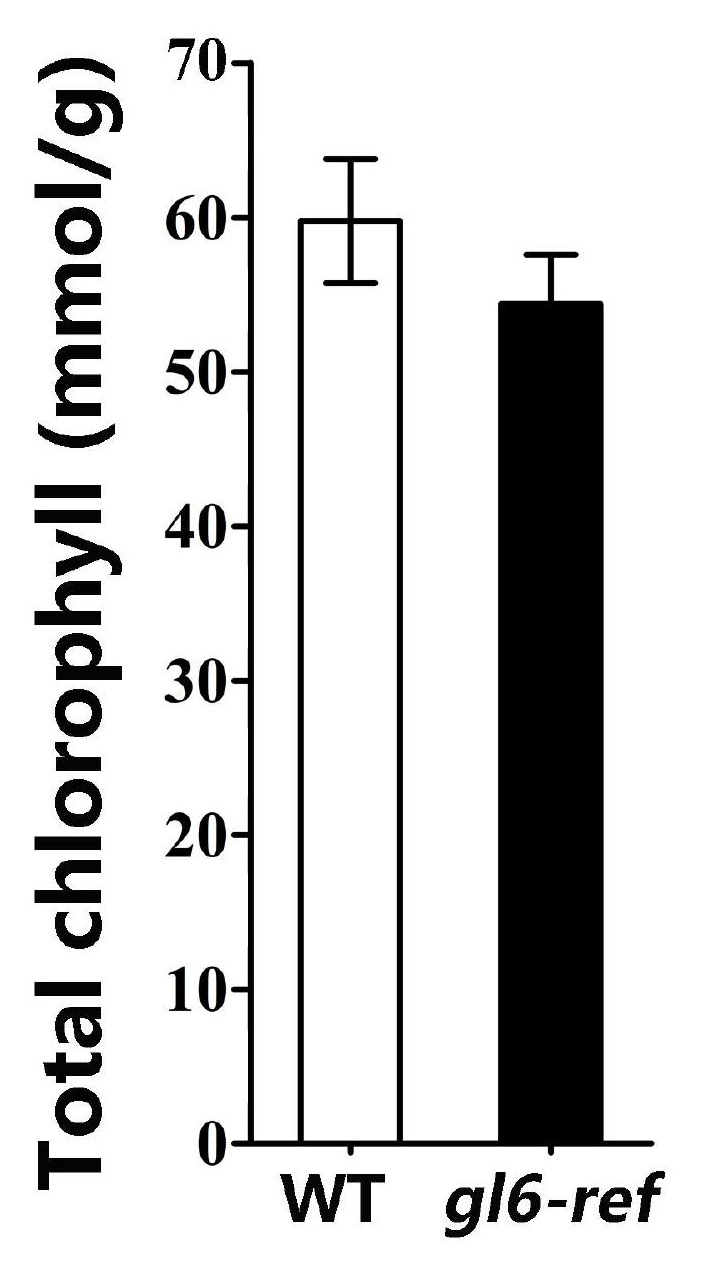

(b)

\section{(d)}
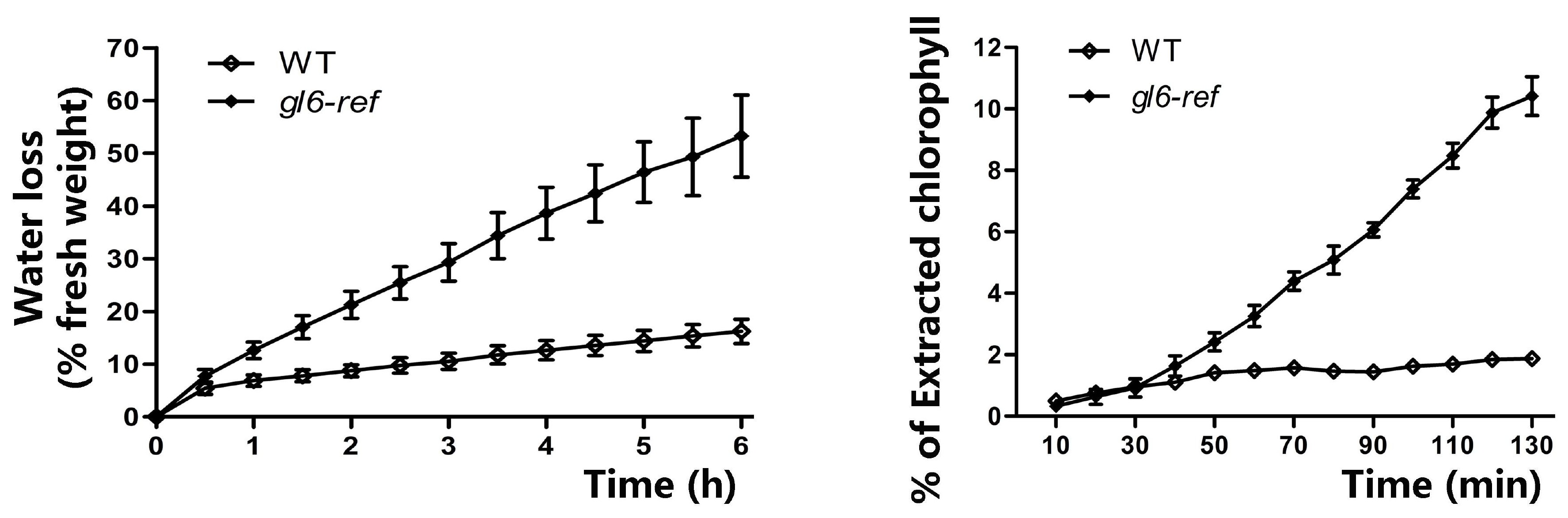
(a)



(e)



(b)

(c)

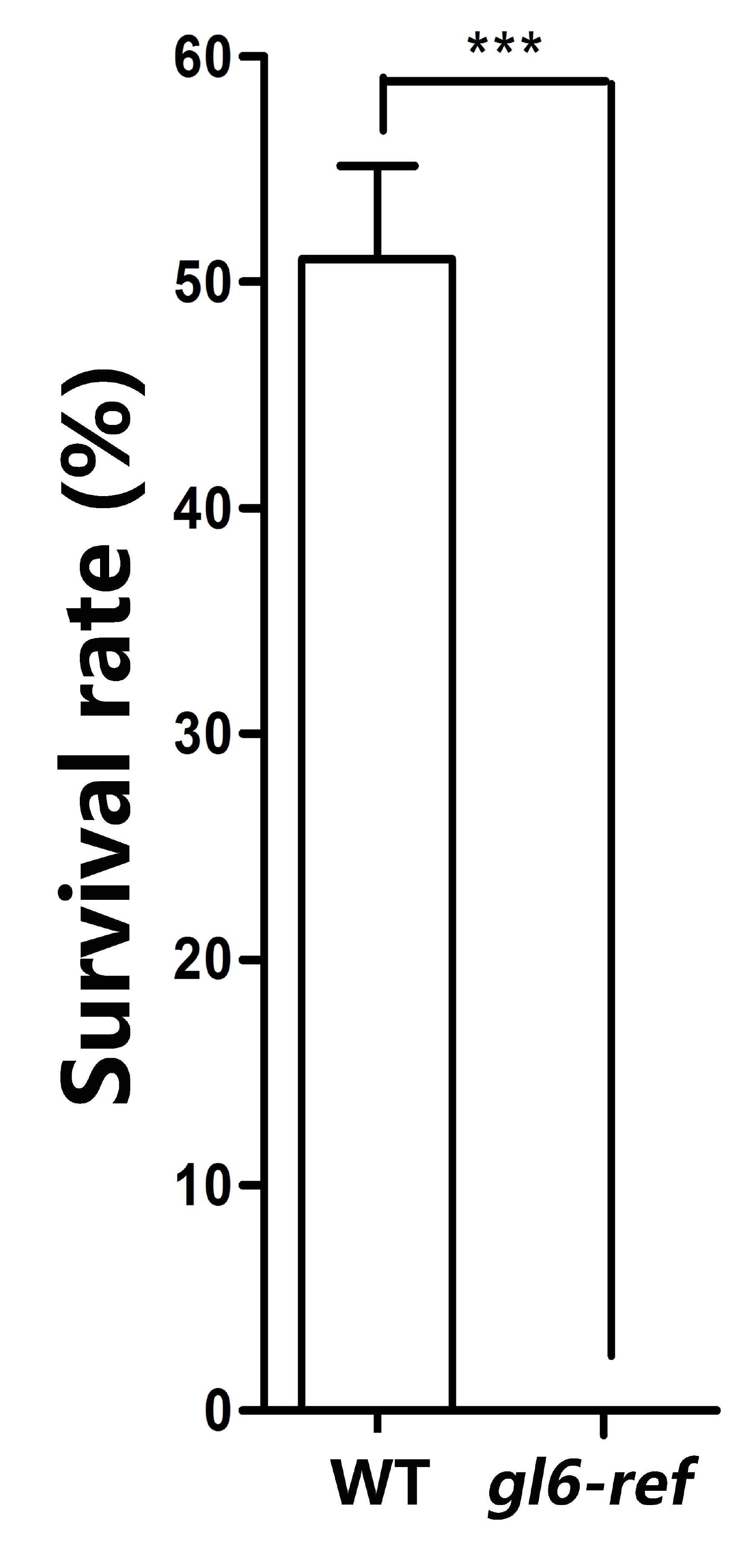

(f)

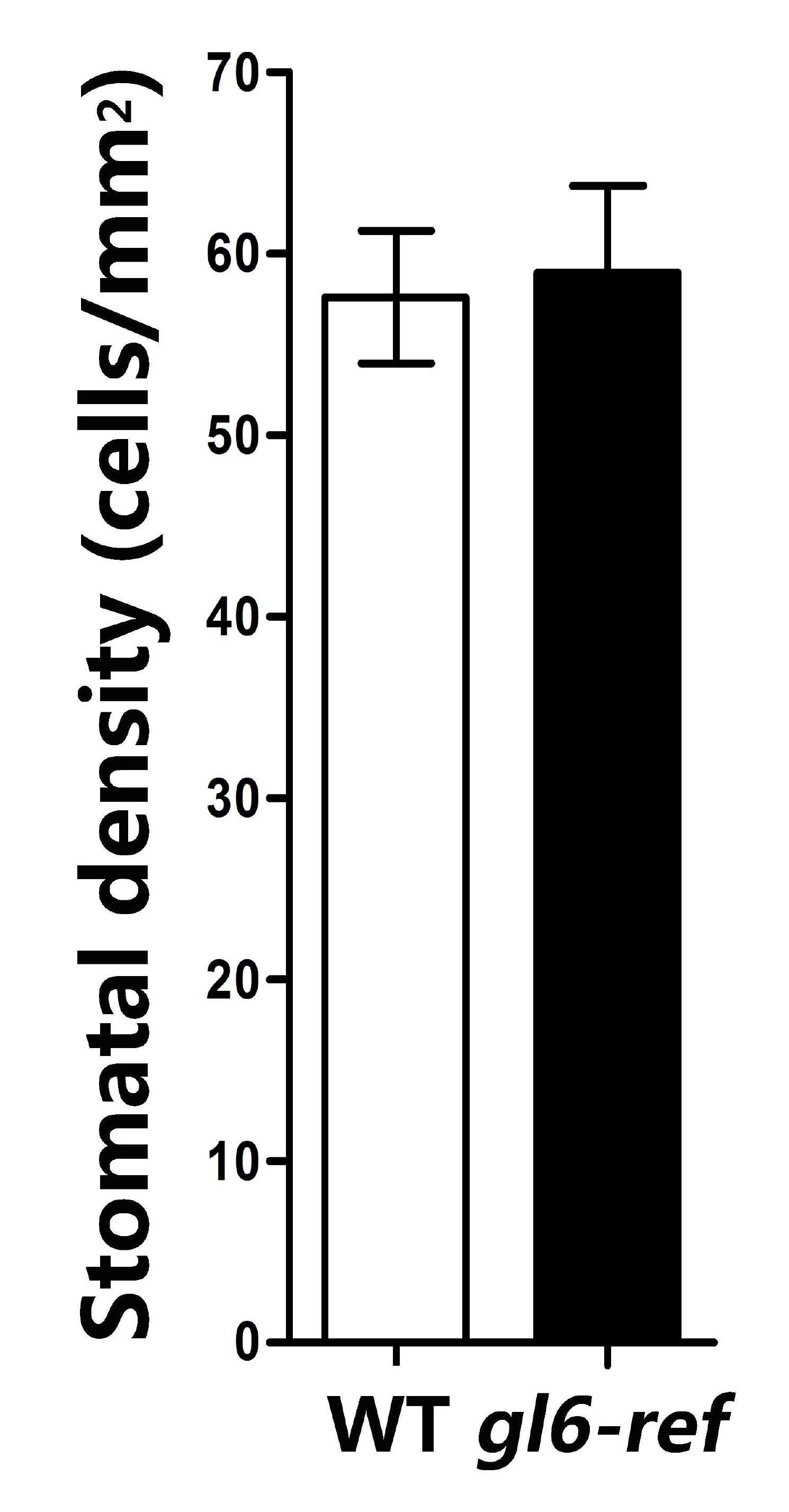

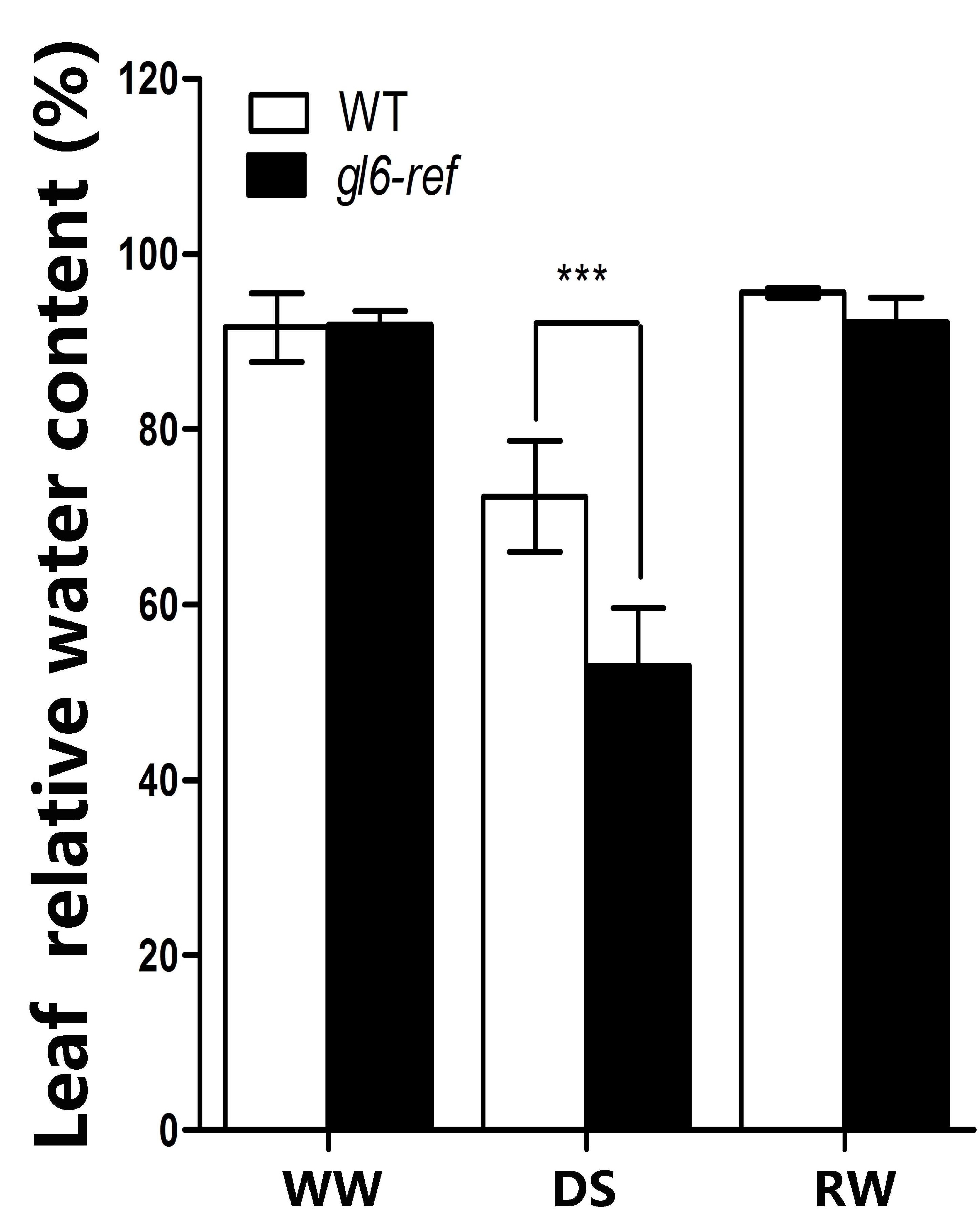

(g)

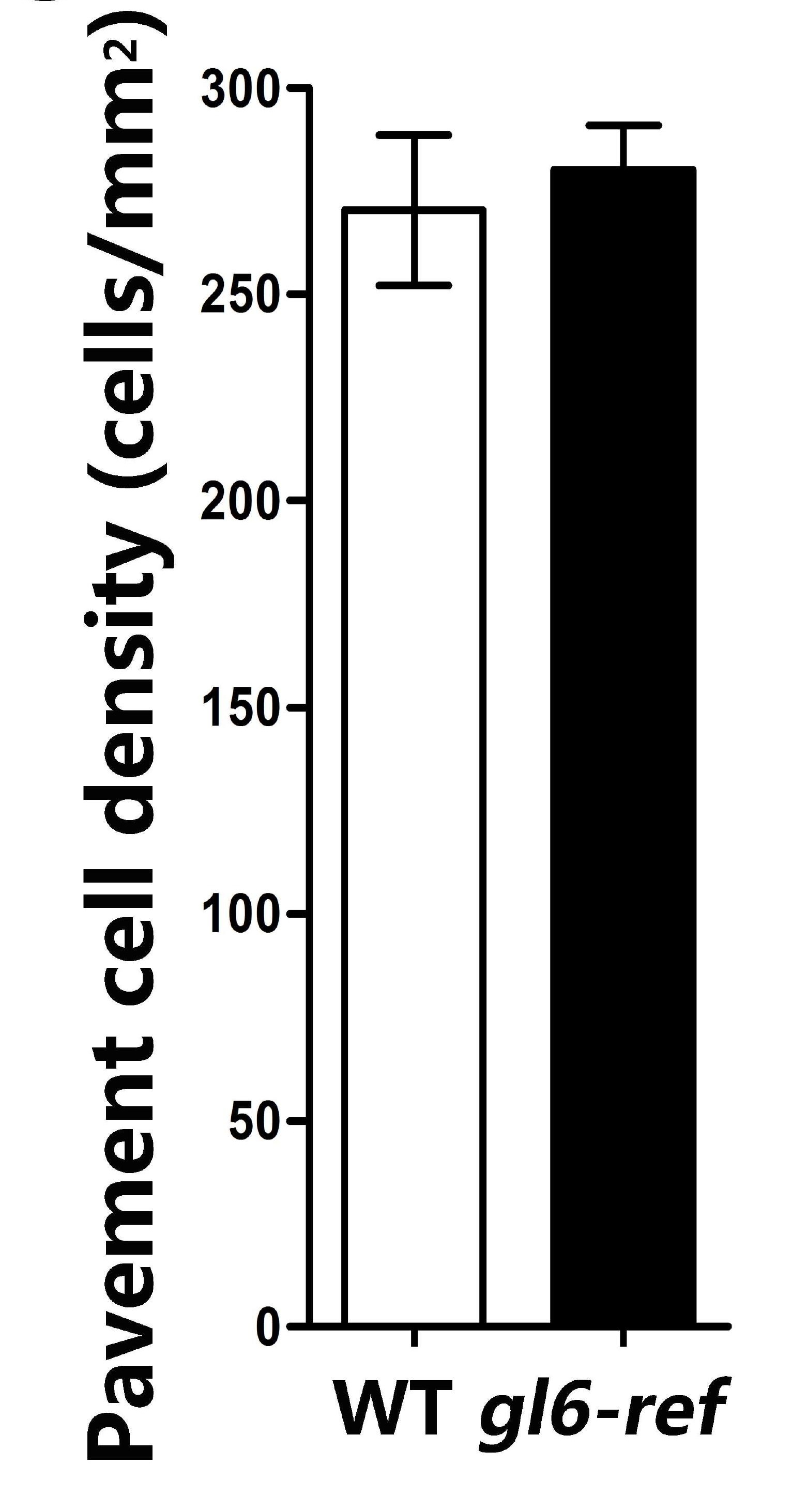

(d)

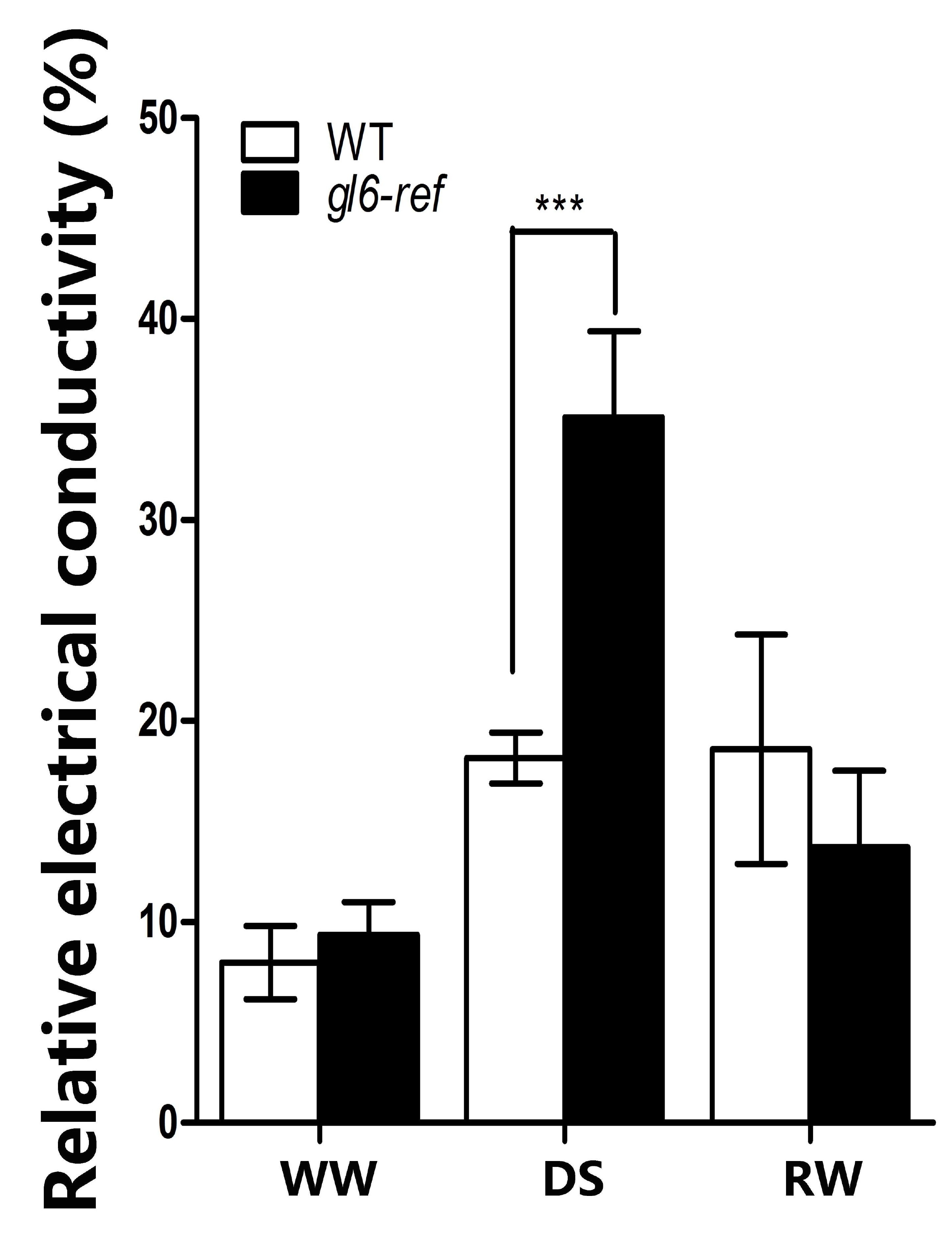

(h)

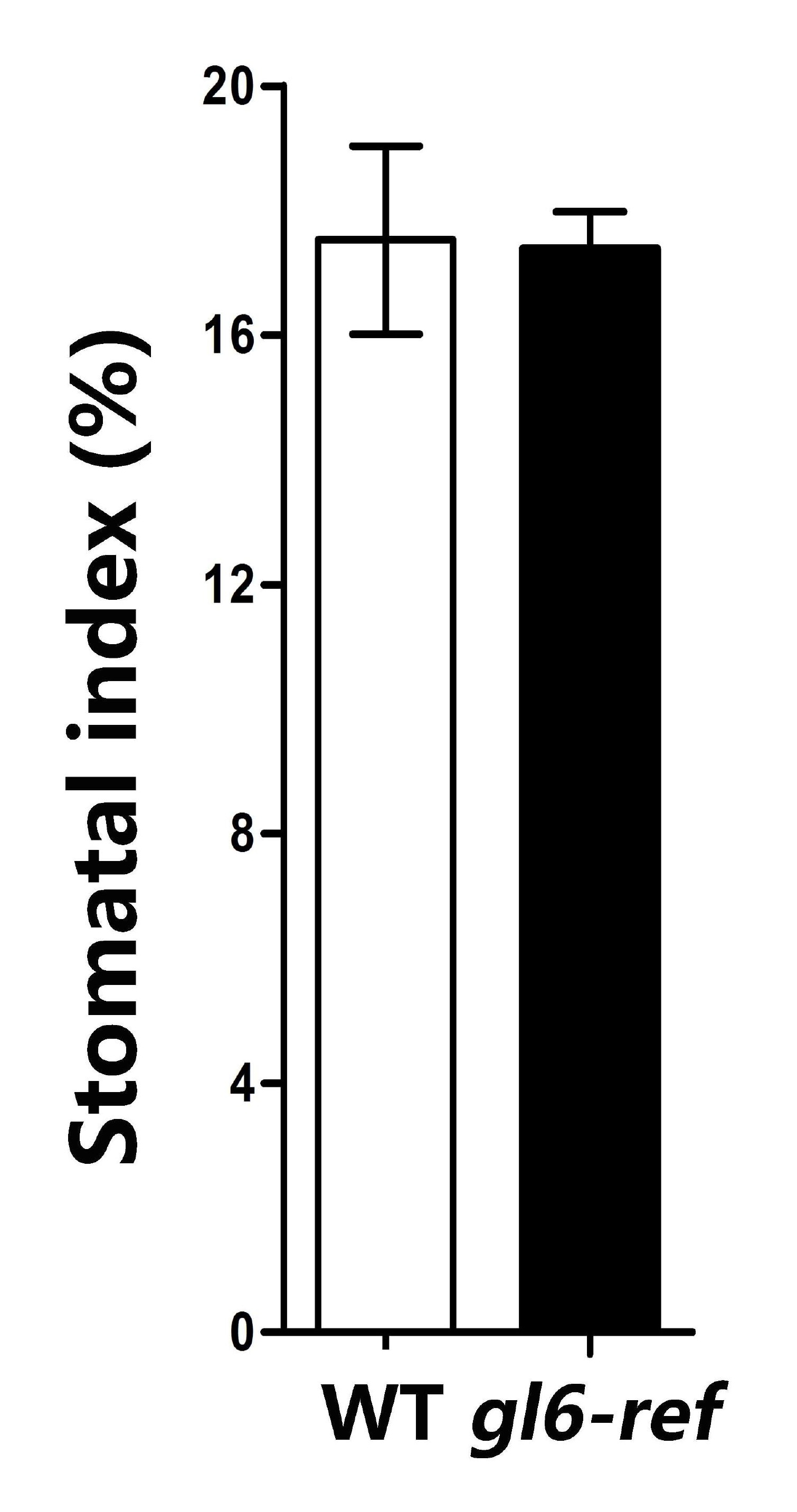




\section{(a)}

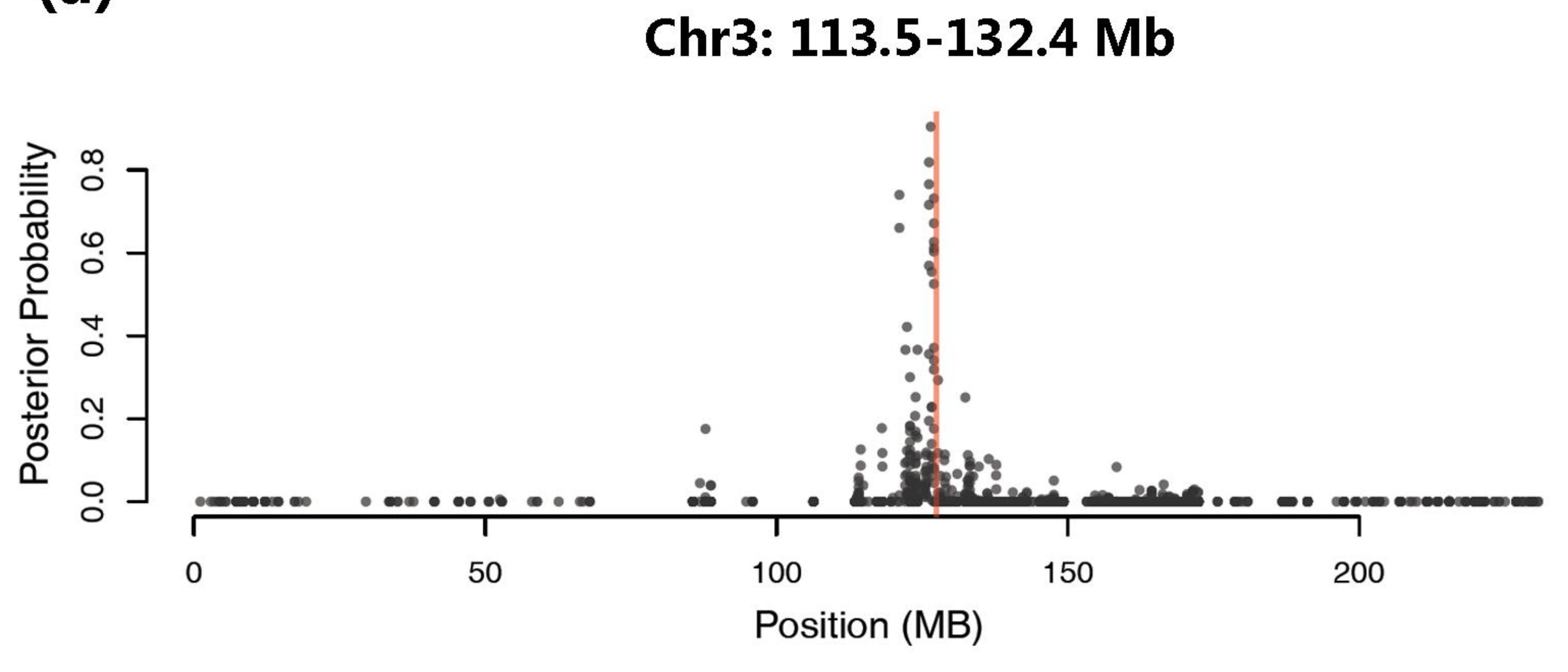

(b)

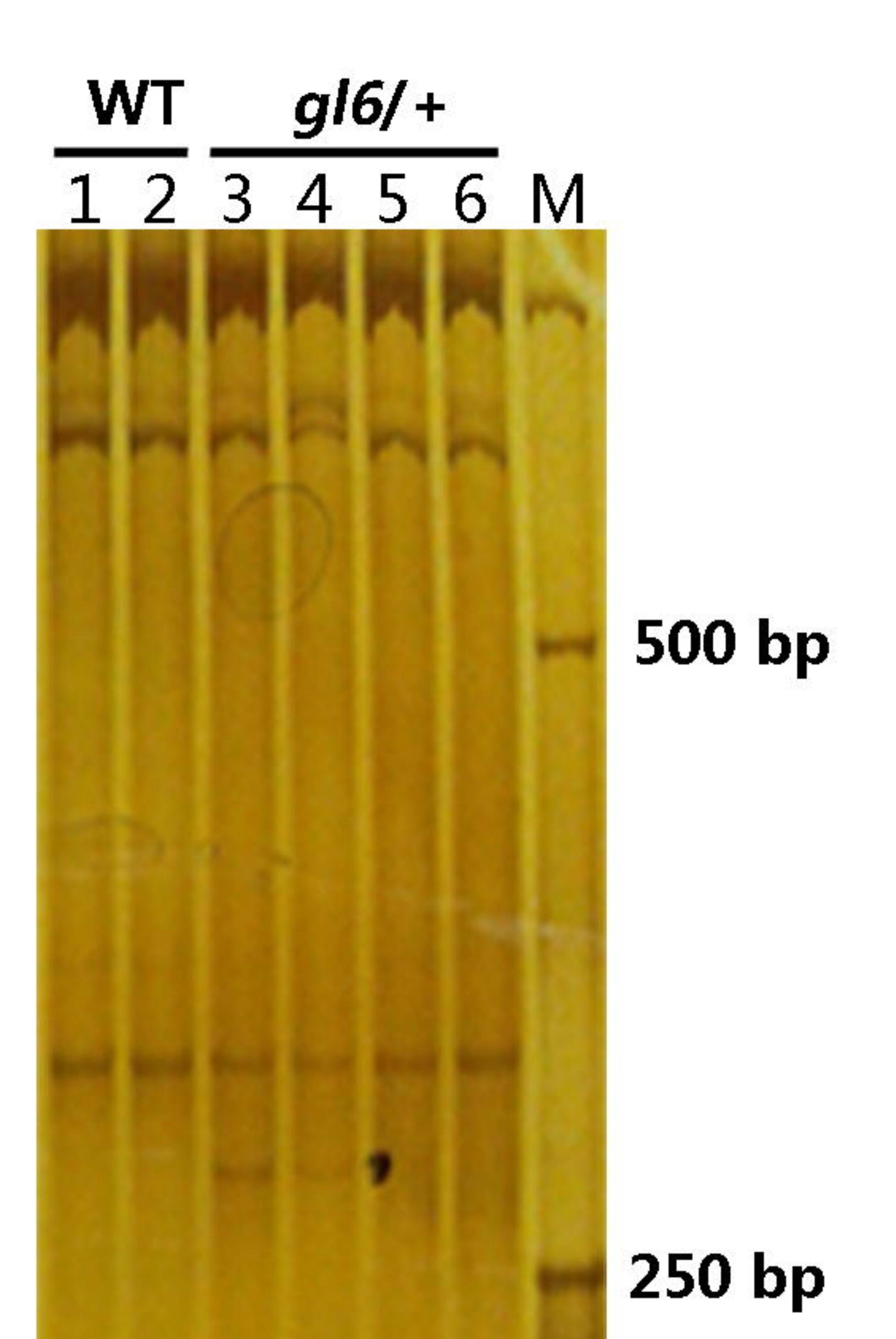

(c)

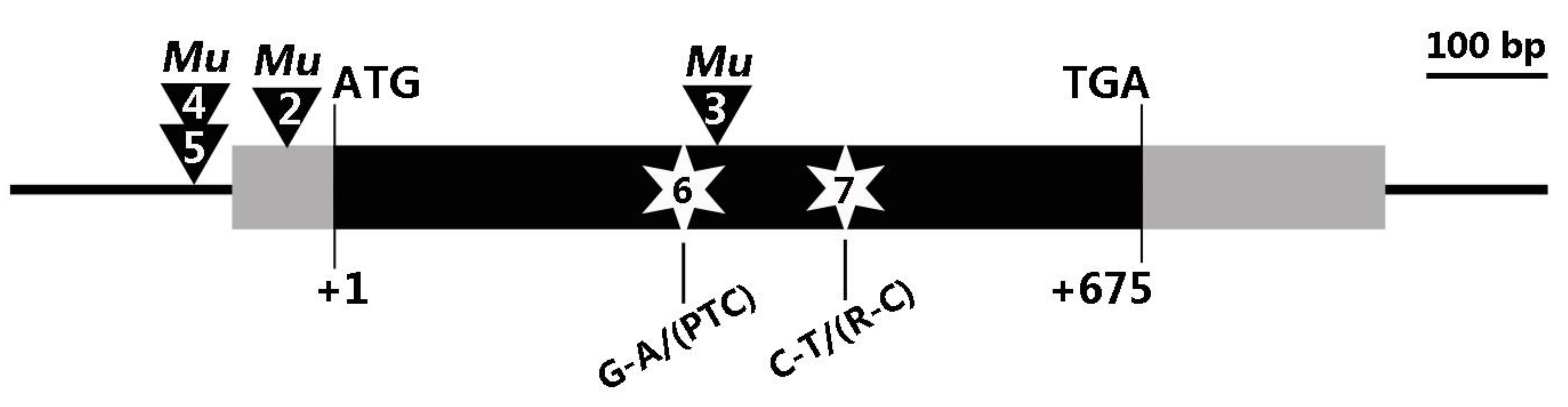

(d)

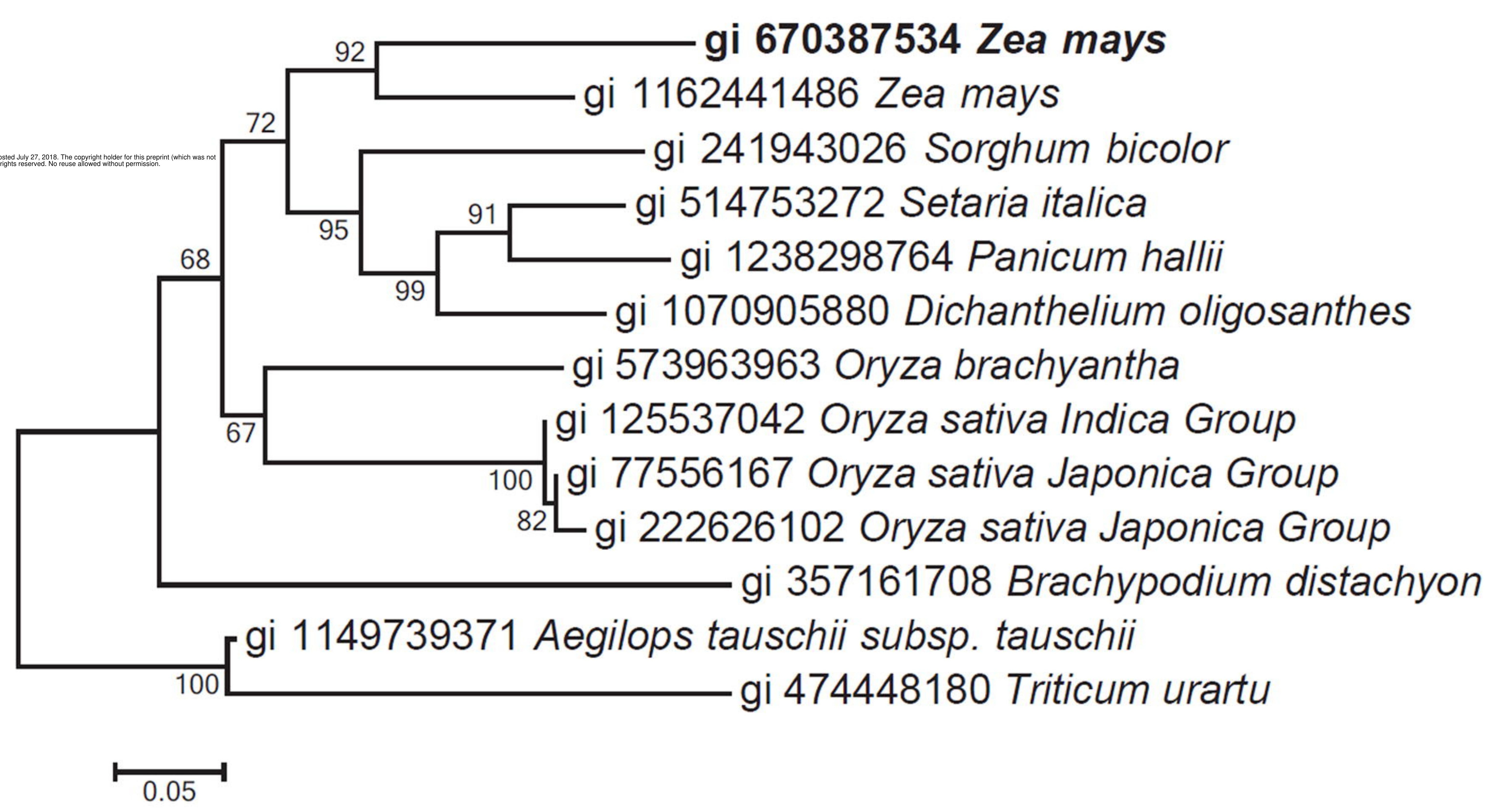


(a)

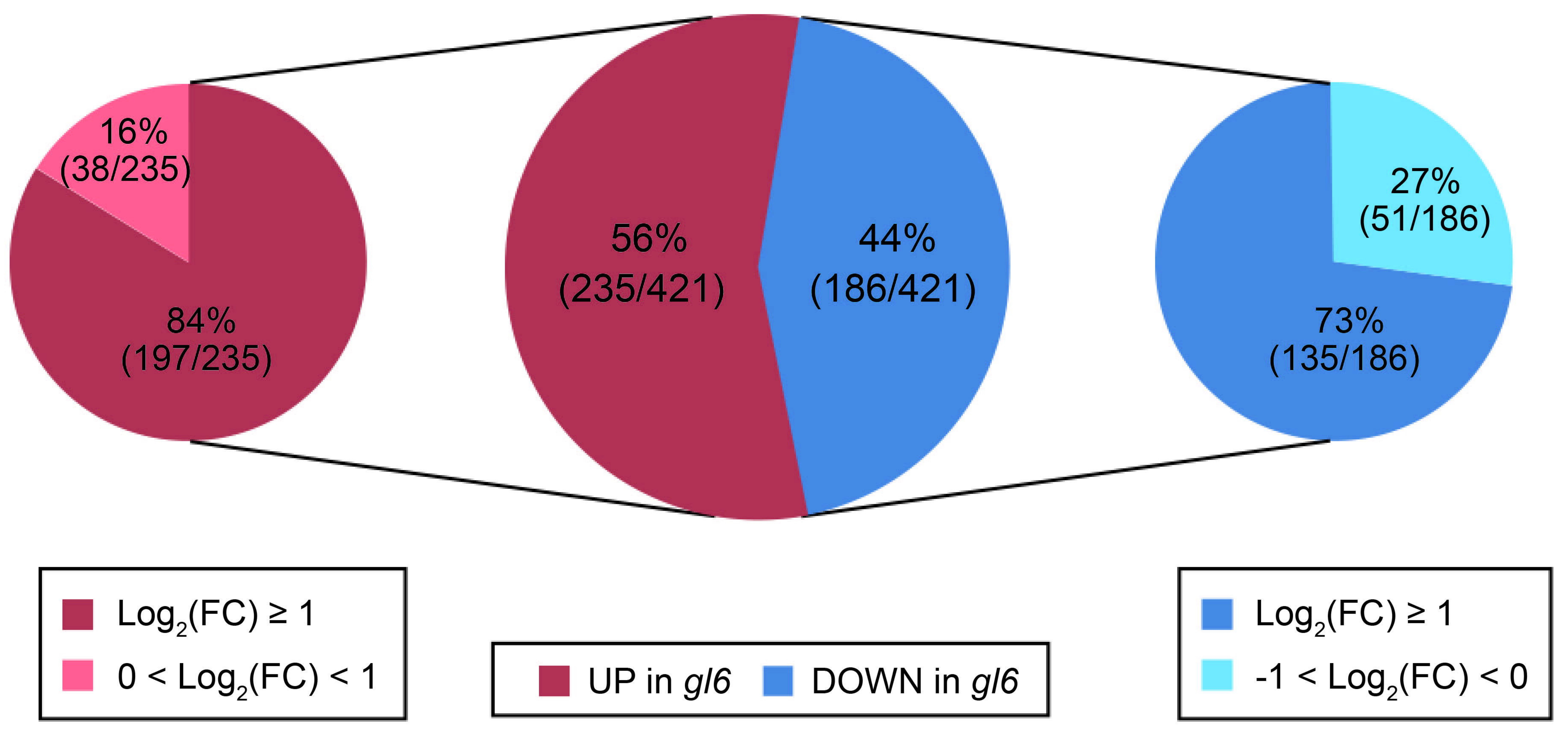

(b)



